\title{
Classification, substrate specificity and structural features of D-2-hydroxyacid dehydrogenases: 2HADH knowledgebase
}

\author{
Dorota Matelska ${ }^{1,2}$, Ivan G. Shabalin ${ }^{1,3}$, Jagoda Jabłońska², Marcin J. Domagalski ${ }^{1,3}$, Jan Kutner ${ }^{1,4}$,
} Krzysztof Ginalski $2^{2^{*}}$ and Wladek Minor ${ }^{1,3,5^{*}}$

\begin{abstract}
Background: The family of D-isomer specific 2-hydroxyacid dehydrogenases (2HADHs) contains a wide range of oxidoreductases with various metabolic roles as well as biotechnological applications. Despite a vast amount of biochemical and structural data for various representatives of the family, the long and complex evolution and broad sequence diversity hinder functional annotations for uncharacterized members.

Results: We report an in-depth phylogenetic analysis, followed by mapping of available biochemical and structural data on the reconstructed phylogenetic tree. The analysis suggests that some subfamilies comprising enzymes with similar yet broad substrate specificity profiles diverged early in the evolution of $2 \mathrm{HADH}$. Based on the phylogenetic tree, we present a revised classification of the family that comprises 22 subfamilies, including 13 new subfamilies not studied biochemically. We summarize characteristics of the nine biochemically studied subfamilies by aggregating all available sequence, biochemical, and structural data, providing comprehensive descriptions of the active site, cofactor-binding residues, and potential roles of specific structural regions in substrate recognition. In addition, we concisely present our analysis as an online 2HADH enzymes knowledgebase.

Conclusions: The knowledgebase enables navigation over the 2HADHs classification, search through collected data, and functional predictions of uncharacterized 2HADHs. Future characterization of the new subfamilies may result in discoveries of enzymes with novel metabolic roles and with properties beneficial for biotechnological applications.
\end{abstract}

Keywords: D-isomer specific 2-hydroxyacid dehydrogenases, Substrate specificity, Sequence-structure-function relationship, Substrate promiscuity, Molecular evolution

\section{Background}

D-2-hydroxyacid dehydrogenases (2HADHs) constitute a widespread family of oxidoreductases, catalyzing the stereospecific, reversible reduction of 2-keto acids to the corresponding 2-hydroxy acids by the simultaneous oxidation of nicotinamide adenine dinucleotide $\left(\mathrm{NAD}^{+}\right)$:

\footnotetext{
*Correspondence: k.ginalski@cent.uw.edu.pl; wladek@iwonka.med.virginia.edu 'Department of Molecular Physiology and Biological Physics, University of Virginia, 1340 Jefferson Park Avenue, Charlottesville, VA 22908, USA ${ }^{2}$ Laboratory of Bioinformatics and Systems Biology, Centre of New Technologies, University of Warsaw, Zwirki i Wigury 93, 02-089 Warsaw, Poland

Full list of author information is available at the end of the article
}

$$
\begin{aligned}
& \mathrm{R}-\mathrm{CO}-\mathrm{COOH}+\mathrm{NAD}(\mathrm{P}) \mathrm{H} \\
& \quad+\mathrm{H}^{+} \leftrightharpoons \mathrm{R}-\mathrm{CH}(\mathrm{OH})-\mathrm{COOH}+\mathrm{NAD}(\mathrm{P})^{+} .
\end{aligned}
$$

2HADHs can act either as reductases or dehydrogenases, use $\mathrm{NADP}(\mathrm{H})$ or $\mathrm{NAD}(\mathrm{H})$ as a cofactor, and possess varied substrate specificities. Due to their diversity of accepted substrates, the enzymes are implicated in different cellular processes, e.g., antibiotic resistance [1], photorespiration [2], or anaerobic glycolysis [3]. In humans, glyoxylate reductase (GRHPR) plays a critical role in the removal of the metabolic by-product glyoxylate from the liver [4]. Mutations in the GRHPR gene were found to cause primary hyperoxaluria type II, a rare disease characterized by endogenous overproduction of oxalate [4]. 
Most sequenced genomes encode multiple 2HADH paralogs. For example, the a-proteobacterium Sinorhizobium meliloti has 16 paralogs, Arabidopsis thaliana has nine, Escherichia coli has five, and the human genome has four. Unfortunately, the exact biological function of the majority of these proteins is unknown because functional annotations of $2 \mathrm{HADHs}$ in protein databases rely on activities obtained for a small subset of selected substrates or on annotations available for the closest characterized homologs. Despite previous efforts [5-7], there is no consistent and comprehensive classification of $2 \mathrm{HADHs}$ into subfamilies. Moreover, no systematic studies show to what extent properties of studied members can be inter- or extrapolated, hindering assignment of biological processes and substrates. Thus, it is often difficult to predict the type of processes that uncharacterized 2HADH members are associated with. Better predictions and annotations would be particularly helpful for studies of medically relevant organisms, which often have several $2 \mathrm{HADH}$ enzymes with unclear functions. In addition, they will help to discover desired enzymes of potential biotechnological applications among a large number of environmental sequences collected from metagenomic samples.

Beyond their multiple cellular functions, 2HADHs have already been shown to possess a range of biotechnology applications. Enantiomerically pure 2-hydroxy acids are versatile building blocks for the synthesis of a variety of significant chiral compounds, which can be used as antimicrobial compounds [8], antitumour antibiotics [9], biodegradable polymers [9] or angiotensin-converting inhibitors [10]. As some 2HADHs can reduce a broad spectrum of 2-keto acids with high efficiency, they are used in systems for highly stereoselective production of selected chiral $\alpha$-hydroxy carboxylic acids [11, 12]. Furthermore, formate dehydrogenase is used for efficient $\mathrm{NADH}$ regeneration in bioreduction systems [13], stimulation of certain metabolic pathways on a cellular level [14], and reduction of the atmospheric $\mathrm{CO}_{2}$ level [15]. Nevertheless, despite of the amount of biochemical, structural, and genomic data, finding or engineering stable and efficient enzymes for particular biotechnological processes have been difficult. Comprehensive classification of the family will help identification of highly efficient and thermodynamically stable enzymes for selected biotechnological processes, and better understanding of functional roles of different structural regions will guide rational design of such biocatalysts.

To better guide functional predictions, rational design, and new applications of these highly important enzymes, we analyzed biochemical and structural information available for $2 \mathrm{HADH}$ members in the light of their evolution. We systematically describe the active site, cofactor-binding residues, and potential roles of specific structural regions in substrate recognition for all the nine biochemically studied subfamilies. Furthermore, we provide a web-based knowledgebase to facilitate functional annotation of uncharacterized members and guide finding of enzymes with particular biochemical characteristics.

\section{Results}

\section{A high-quality phylogenetic tree of the 2HADH family}

We calculated multiple phylogenetic trees in various ways (using neighbor-joining and maximum-likelihood approaches) and used nodes with high split support values (i.e., greater than 0.5) to assign sequences to subfamilies. Here, we define "subfamily" as a group of proteins that appear consistently as a clade in all the phylogenetic trees, which presumably share a similar function. Although low support values for the bifurcations close to the mid-point root indicate uncertainty of the path of the early evolution of the 2HADH family, the major subfamilies appear consistently as separate clades in the computed trees (Fig. 1). The 22 identified subfamilies include nine in which at least one member has been studied biochemically. Five of these subfamilies appeared in the previous classification [5]: 3-phosphoglycerate dehydrogenases (SERA), formate dehydrogenases (FDH), C-terminal binding proteins (CTBP), 4-phosphoerythronate dehydrogenase (PDXB), and D-lactate dehydrogenases (LDHD).

Due to little sequence similarity among distant $2 \mathrm{HADH}$ members (as noted earlier [16, 17]), noteworthy differences in subfamily classification may arise from the use of alternative methodologies for phylogenetic reconstruction. Notably, three subfamilies were classified into a single glyoxylate/hydroxypyruvate reductase (GHPR) cluster in the earlier neighbor-joining phylogenetic trees based on ClustalW sequence alignments $[5,6]$. Here, these subfamilies are referred to as: glyoxylate/hydroxypyruvate reductases A (GHRA; including GhrA from E. coli, GHRA ECOLI), glyoxylate/hydroxypyruvate reductases B (GHRB; including GhrB from E. coli, GHRB_ECOLI, and PtxD from Pseudomonas stutzeri, PTXD_PSEST) and broad-substrate-specificity dehydrogenases (DDH; including DDH from Haloferax mediterranei, DDH_HALMT). In all reconstructed trees, DDH and GHRA appear as closely related, yet separated, clades. Similarly, the polyphyletic origin of the GHRB subfamily and the clade encompassing GHRA and DDH subfamilies is supported in all reconstructed trees. Although in previous studies some GHRA and GHRB members showed similar substrate profiles and were classified as one group [5], in our analyses, they consistently appear as distantly related clades, separated early in the evolution of the $2 \mathrm{HADH}$ family (Fig. 1). Within GHRB, we also found a significant premise for a horizontal gene transfer from bacteria to plants, potentially occurred after early diversification of 


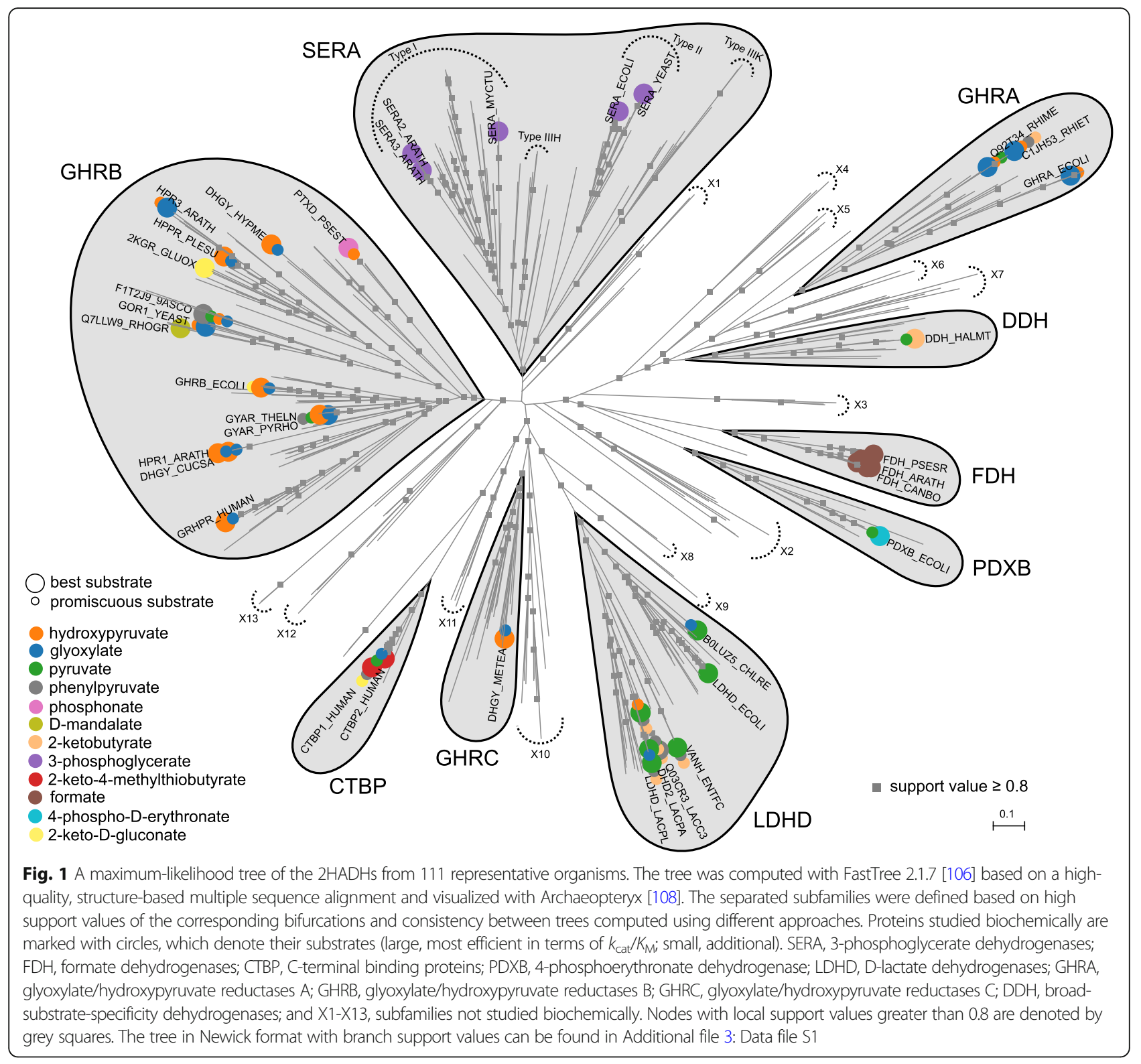

mesangiosperms [18] (elaborated in Additional file 1: Supplementary Results).

Besides GHRA, GHRB, and DDH, a fourth clade includes an enzyme previously shown to act as a hydroxypyruvate/glyoxylate reductase. HprA from the facultative methylotroph Methylobacterium extorquens (DHGY_METEA) plays a central role in carbon assimilation, as it converts hydroxypyruvate to glycerate in a critical step of the serine cycle [19]. The corresponding subfamily, which we name glyoxylate/hydroxypyruvate reductases C (GHRC), comprises bacteria from various phyla as well as a methanogenic archaeon, Methanococcus maripaludis, and has not been featured in previous classifications.

Along with the nine studied subfamilies, 13 additional clades not studied biochemically (X1-X13), including eight with representatives with a determined 3D structure (Additional file 2: Figure S1), could be defined with high support values (Additional file 3: Data file S1). Single long branches were left outside the classification; however, if more sequences were added, they could constitute additional clades.

\section{Substrate specificity of the 2HADH enzymes}

To systematically describe the properties of the $2 \mathrm{HADH}$ subfamilies, we collected enzymatic parameters for the characterized representatives from the available literature (Additional file 4: Table S1). The collected data include 77 enzyme-substrate pairs with determined catalytic efficiency, defined as $k_{\text {cat }} / K_{\mathrm{M}}$, based on which 14 compounds are 'best' substrates for at least one 
enzyme. Although 2HADHs were usually studied against a just a few substrates, most of them can be considered as promiscuous enzymes based on the collected data. The only exception constitutes FDHs, for which no substrates other than formate were determined so far; FDHs work through a different reaction mechanism, without typical stages of acid-base catalysis [20, 21]. Cumulatively, the $2 \mathrm{HADH}$ proteins are versatile catalysts in vitro-in total, they were shown to accept 33 compounds with either cofactor (Additional file 4: Table S1). The median $k_{\mathrm{cat}} / K_{\mathrm{M}}$ for the 'best' substrates is $1.45 \times 10^{5}$ $\mathrm{M}^{-1} \mathrm{~s}^{-1}$ (Additional file 5: Figure S2), thus 2HADHs can be considered as moderately efficient catalysts, as compared to global trends for enzymes [22]. Interestingly, the two subfamilies most conserved regarding sequence and function, FDH and CTBP, comprise the least efficient catalysts, characterized by $k_{\text {cat }} / K_{\mathrm{M}}$ of $10^{2}-10^{3}$ $\mathrm{M}^{-1} \mathrm{~s}^{-1}$ and $\sim 10^{3} \mathrm{M}^{-1} \mathrm{~s}^{-1}$, respectively (Additional file 5: Figure S2). On the other hand, some of the most divergent subfamilies, GHRB and LDHD, encompass the most promiscuous and efficient enzymes.

In the studied in vitro conditions, most of the $2 \mathrm{HADH}$ subfamilies comprise members acting as reductases towards 2-keto acids, with a simultaneous oxidation of $\mathrm{NADH}$ or NADPH. Only three subfamilies contain representatives natively working as dehydrogenases, i.e., towards formate (FDH), 3-phosphoglycerate (SERA) and 4-phospho-D-erythronate (PDXB). In addition, two dehydrogenases were described in the highly heterogeneous GHRB cluster, i.e., phosphonate dehydrogenase from P. stutzeri [23] and D-mandelate dehydrogenase from Rhodotorula graminis [24]; also, the only characterized member of the GHRC subfamily was shown to possess glycerate dehydrogenase activity [19]. Unlike reductases, which have preferences for either NADH or $\mathrm{NADPH}$, almost all wild-type dehydrogenases efficiently employ only $\mathrm{NAD}^{+}$as a cofactor, which is expected given the typical redox state of a cell [20]. However, some formate dehydrogenases have been shown to possess dual cofactor specificity (i.e., working with $\mathrm{NAD}^{+}$ and $\left.\mathrm{NADP}^{+}\right)[19,25]$.

In general, the data suggest that well-evolved enzyme-substrate interactions are rather rare among 2HADHs. Median affinity to the "best" (i.e., catalyzed with the highest efficiency) substrates, approximated as $K_{\mathrm{M}}$ (or $K_{1 / 2}$ in case of non-Michaelis-Menten behavior), is lower than the average affinity for metabolic enzymes in general $\left(K_{\mathrm{M}}\right.$ of $600 \mu \mathrm{M}$ against $130 \mu \mathrm{M}[22,26]$, respectively). Only a few $2 \mathrm{HADHs}$ display higher affinity (compared to the global average) for their native substrates. Most of these are promiscuous 2HADH enzymes, with $E$. coli $\mathrm{PdxB}$ in the extremum $\left(K_{\mathrm{M}}\right.$ of $2.9 \mu \mathrm{M}$, Additional file 5: Figure S2) [27]. As described for other enzyme families [26], a substrate considered as the physiological or most efficient in vitro in one subfamily is often secondary in other subfamilies (Fig. 1). In $2 \mathrm{HADHs}$, glyoxylate, hydroxypyruvate, and pyruvate recurrently appear as accepted substrates in most subfamilies.

\section{Analysis of crystal structures}

Besides kinetics, a wealth of structural data is also available for 2HADH enzymes. Out of the 22 defined subfamilies, 16 contain representative proteins with solved crystal structures (Additional file 2: Figure S1). Among the $1212 \mathrm{HADH}$ structures available in the PDB, 40 were solved with both a cofactor and a ligand bound in the active site (Additional file 6: Table S2). Nevertheless, almost half of the structures have not been discussed in the literature (as indicated by the lack of a linked reference publication in the PDB). Several subfamilies (DDH, GHRC, X4, X6, X7, X9, X10, X12, and X13) have representative structures available in the $\mathrm{PDB}$, but not a single one was presented in the scientific literature.

2HADHs usually act as homodimers, where each monomer is composed of two domains: a cofactor-binding domain with a classical NAD(P)-binding Rossmann fold, and a substrate-binding (or catalytic) domain with a modified (flavodoxin-like) Rossmann fold [28] (Fig. 2). The cofactor-binding domain is embedded in the substrate-binding domain and characterized by a more conserved sequence. The active site is located in the cleft formed between the two domains and is built mainly with residues from the cofactor-binding domain. According to solved crystal structures of apo and holo forms, 2HADHs can exist in either "open" and "closed" conformational states. Transition from the open to the closed conformation is essential for the formation of the enzyme active site and for catalysis [29]. Generally, crystal structures of $2 \mathrm{HADHs}$ without the cofactor bound display the open conformation, and holo forms display the closed conformation. However, there are a few exceptions to that general trend, which are likely caused by compounds present in the crystallization cocktails (e.g., sulfates) and different crystal environments [30]. The general consensus is that the $2 \mathrm{HADH}$ enzymes are in a dynamic equilibrium between the open and closed states and that the binding of cofactor shifts the equilibrium towards the closed state [29]. Because the substrate binds to residues from both domains, its binding is likely to contribute to shifting the equilibrium towards the closed state.

The structure-based alignment of representative sequences shows conservation of several residues (Additional file 7: Figure S3), suggesting a crucial role for these amino acids across the whole family. Some of them are well known to perform crucial functions, yet others were not previously discussed in the literature (e.g., 


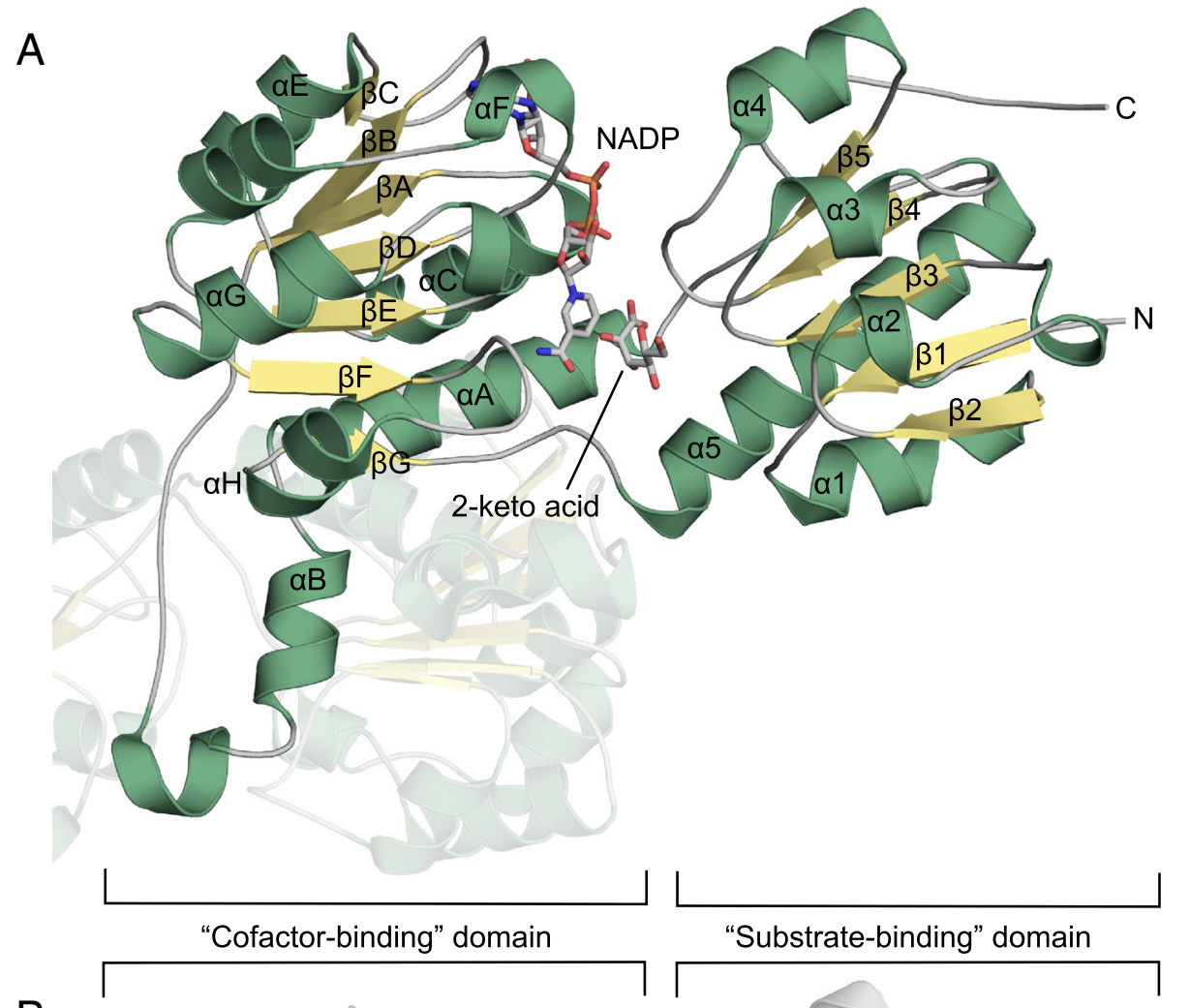

B

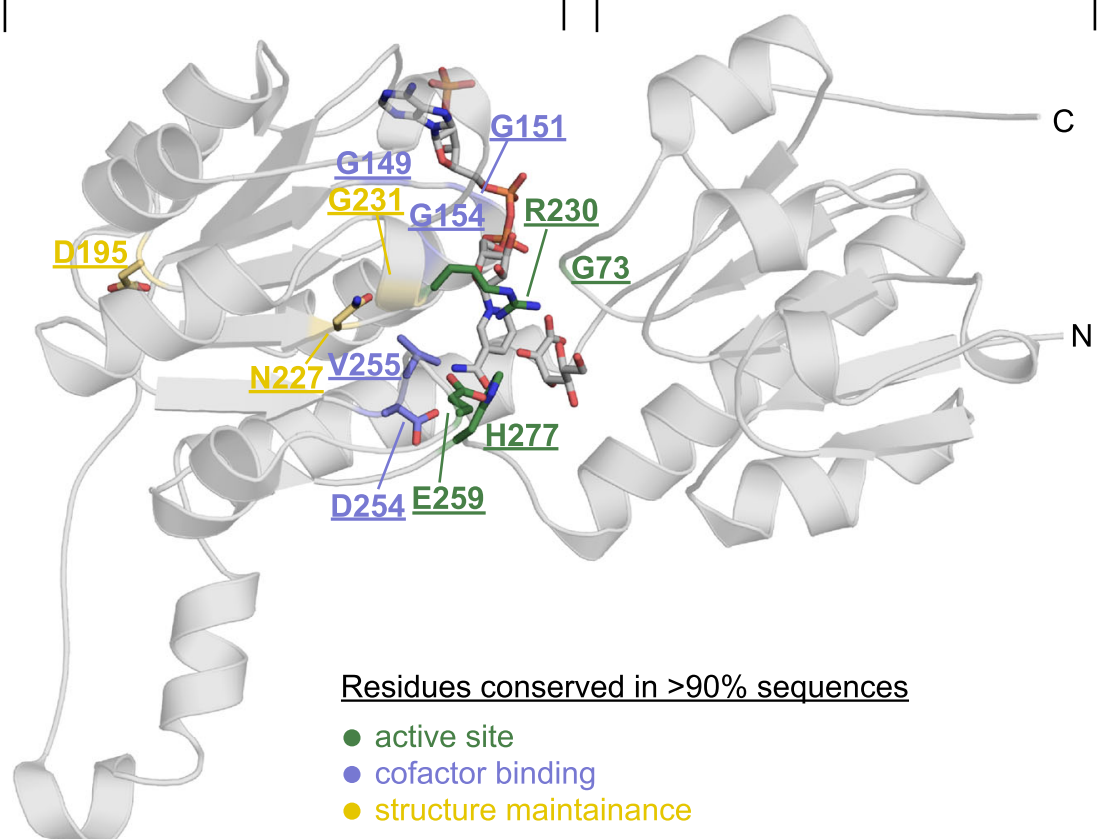

Fig. 2 Crystal structure of a 2HADH from Sinorhizobium meliloti (PDB ID: 5V7n) complexed with a cofactor (NADP ${ }^{+}$) and a reaction product (2-keto-Dgluconic acid). Cofactor-binding and substrate-binding domains are indicated by brackets. $\mathbf{a}$, Secondary structure elements are labeled; the other subunit of the dimer is translucent. $\mathbf{b}$, Highly conserved residues (>90\% in all 2HADH sequences) are labeled

Val72, Gly73, Asn227, Gly229, and Gly231, see below). The importance of some of these residues could only be determined by family-wide sequence comparison, as opposed to analyses of single structures, which only highlight important features of a particular enzyme. We divide the functions of highly conserved residues (>90\% conservation across all $2 \mathrm{HADH}$ sequences) into three categories-residues binding the cofactor, contributing to catalysis, and maintaining overall structure (Fig. 2). In the following analysis, the sequence numbering is 
according to a representative enzyme from the GHRB subfamily, Q92LZ4_RHIME (PDB ID: 5v7n).

\section{Residues binding the cofactor}

Among the residues crucial for cofactor-binding, a highly conserved pyrophosphate-binding GXXGXGXXG motif (residues 146-154 in Q92LZ4_RHIME) is common for Rossmann-fold dinucleotide-binding proteins. The motif is located in the region connecting the first strand of the $\beta$-sheet to the $\alpha$-helix of the Rossmann fold (i.e., $\beta \mathrm{A}-\alpha \mathrm{C}$ ). It contributes to the structural arrangement of the pyrophosphate bridge of the cofactor by assuring nearly optimal dihedral angles [31]. Although mutations of the glycine residues result in a drastic loss of enzymatic activity [32], neither of them are totally conserved among all 2HADH sequences (Fig. 3). It was shown that an Ala-to-Gly mutation improves protein thermal stability and decreases $K_{\mathrm{M}}$ towards NADH [33]. Two other conserved residues, aspartate (Asp254) and valine (Val255) contribute to binding of the pyridine ring of the cofactor [34, 35].

The specificity towards the cofactor $[\mathrm{NAD}(\mathrm{H})$ vs. $\operatorname{NADP}(H)]$ is effectively defined by the residues located in the $\beta \mathrm{B}-\alpha \mathrm{D}$ loop at the pocket that binds adenine and ribose moieties of the cofactor (Fig. 3). Five of the nine biochemically characterized subfamilies (CTBP, FDH, LDHD, PDXB, and SERA) have a highly conserved aspartate residue in this region, corresponding with a preference for NADH [36]. Many of GHRA enzymes have the characteristic motif [ST]R[ST]X[RK] in the same $\beta \mathrm{B}-\alpha \mathrm{D}$ loop - a conserved sequence fingerprint corresponding with specificity towards $\mathrm{NADP}(\mathrm{H})$. Other three biochemically characterized subfamilies do not have highly conserved residues in the region, suggesting varying cofactor specificity within these subfamilies.

\section{Residues of the active site}

The catalytic mechanism for the $\mathrm{NAD}^{+}$-dependent oxidation of 2-hydroxy acids (and the reverse reduction) is dependent on an internal acid-base catalyst, typically histidine [37, 38]. During the oxidation reaction, a hydride ion leaves the $\mathrm{C} 2$ atom of a substrate and attacks the $\mathrm{C} 4$ atom of the $\mathrm{NAD}^{+}$pyridine moiety, and a proton moves from the hydroxy group of the substrate to the histidine of the active site [29]. Together with the histidine, two more residues within the active site-Arg and Glu/Asn-are thought to contribute directly to the reaction and are referred to as the "catalytic triad" [39]. The highly conserved arginine stabilizes and polarizes the bound substrate, whereas the glutamate (or asparagine in formate dehydrogenases) stabilizes the protonated form of the catalytic histidine.

The mode of the substrate binding was subject to long discussions over several years; at least three modes of 2-keto/2-hydroxy acid binding were proposed [29, 34]. As our analysis of crystal structures shows, despite 40 structures being solved with both a cofactor and a ligand bound in the active site, only eight of them represent a

\begin{tabular}{|c|c|c|c|c|c|c|c|c|c|c|c|}
\hline & $\beta 1-\alpha 1$ & $\beta 3-\alpha 3$ & $\beta 4-\alpha 4$ & $\beta 5-\alpha A$ & $\alpha B^{\prime}-\beta A^{\prime}$ & $\beta A-\alpha C$ & $\beta B-\alpha D$ & $\beta E-\alpha G$ & $\beta F-\alpha H$ & $\beta G-\alpha 5$ & $a 5$ \\
\hline CTBP & $D G \quad R D$ & Y HTIT & RIGSGFD & $\begin{array}{l}P \\
P_{\triangle A s}\end{array}$ & BuOS VEQI REV & $\overrightarrow{G E I G G G R Y G}$ & $\widehat{Y D P Y_{L}}$ & 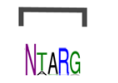 & DVHESEP. & TPHAAllYS $_{\text {S }}$ & EQASEEM \\
\hline DDH & 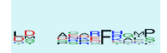 & 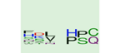 & $\therefore A G \times D$ & $=G \times H$ & $\|_{\text {Baseg }}$ & $v_{y} \forall G_{L} G_{x}[G$ & B & 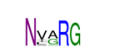 & DVtropep & IPHW & Cren \\
\hline FDH & cy & DPFHPAY & TAGTGSD & Ts:SN & $\|_{\text {Qya }}$ & GTV\&AGRIG & DBB & NTARG & DVVIIEPQP & TPHESGTST & LDAQRARY \\
\hline GHRA & 줄 CEares & & $\therefore$ \&AGVD & B & $\|_{3-1}$ & GTMGGGuG & 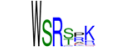 & $N_{\triangle} \in R G$ & DVFEE & $T P H_{2 A} A_{x \times \frac{T}{X}}$ & 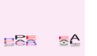 \\
\hline GHRB & 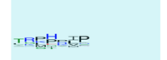 & 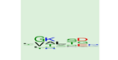 & $=x \in G \times 0$ & $P_{\mathrm{o} X L}$ & $\underline{W} w_{B=x} W_{p e e}$ & $G I=0 G G G I G$ & 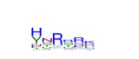 & $N_{ \pm} \mathbb{R} \mathbb{R}_{G}$ & DV ${ }_{E_{E}}, E P$ & PHIESAT & $\in \mathbb{I R} M$ \\
\hline GHRC & LDEXXLيP & I NKK & $Y=A^{\top} G Y N$ & E8 $1 / 5$ & $\|_{\operatorname{mas}}$ & $G \in E G=G \in[G$ & XPBSE & NIARG & DV & TPH:AllAS & EAC RL \\
\hline LDHD & $\triangle B \quad Y E Y Q Y Y$ & $E \mathrm{YNO}$ & $R=A G E N$ & $P_{A} Y_{S}$ & $E_{1}=A$ & GYTGTGKIG & YDexpy & NIRAG & DyYEerE & TPUlpAEYYT & 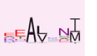 \\
\hline PDXB & DE NM & R SVTKK & TATAGTD & $\mathrm{PGCN}$ & & GTYGXGQVGG & CDPPBA & $N_{A} \subseteq R G$ & DVWUEs:EP & Te्रीLAGY. & LEGKABG \\
\hline SERA & 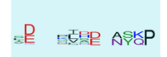 & $R S_{n} T_{5}$ & $R_{A} G_{*} G_{X} \times R_{N}$ & Ped & $\|_{a}$ & GT工 & $y \mathrm{P}_{\mathrm{z}}$ & $N_{8} A B G$ & DVF & IPAPGGAST & $E A Q m X$ \\
\hline & & & & & & $\begin{array}{l}\text { pyrophosphate } \\
\text { binding }\end{array}$ & $\begin{array}{l}\text { NAD/NADP } \\
\text { specificity }\end{array}$ & $\Delta$ & $\underset{\text { talytic triad }}{\Delta}$ & $\Delta$ & \\
\hline \multicolumn{12}{|c|}{$\begin{array}{l}\text { Fig. } 3 \text { Sequence logos of selected regions critical for substrate and cofactor binding in the nine biochemically studied 2HADH subfamilies. The } \\
\text { structure-based alignment was obtained for selected structures with PROMALS3D and used as a seed alignment for other } 2 \text { HADH sequences } \\
\text { from } 111 \text { representative organisms. The sequence logos were generated with WebLogo, showing columns for which in at least one subfamily at } \\
\text { least } 90 \% \text { members possess an amino acid (i.e., with at most 10\% gapped positions). Rectangles with colored backgrounds comprise loops } \\
\text { implicated in substrate specificity. Catalytic triad residues are denoted by red triangles. Sequence logos of the full-length alignments are shown in } \\
\text { Additional file 7: Figure S3 }\end{array}$} \\
\hline
\end{tabular}


true ternary complex-a complex with a reduced cofactor and a reduced substrate, or with an oxidized cofactor and an oxidized substrate (Additional file 6: Table S2). The remaining triple complex structures have an inhibitor, a substrate analog, or a solute bound in the active site and do not provide a complete model of substrate binding (except for FDH, whose substrate is not a 2-hydroxy acid), as discussed earlier [29]. In 2006, the first crystal structure of a true ternary complex of a 2HADH (human GHRB, GRHPR_HUMAN, PDB ID: $2 \mathrm{gcg}$ ) was published [38], demonstrating the interactions between the substrate and catalytic residues within the active site and confirming one of the earlier proposals for the mode of substrate binding (Fig.4). Later, the same mode of substrate binding was observed in all other $2 \mathrm{HADH}$ true ternary complexes with clearly observed electron densities for the ligands: human CTBPs (CTBP1_HUMAN and CTBP2_HUMAN) in complex with $\mathrm{NAD}^{+}$and 4-methylthio-2-oxobutyric acid (PDB ID: 4lce and 4lcj) [40], GHRB from Rhizobium meliloti (Q92LZ4_RHIME) in complex with $\mathrm{NADP}^{+}$and 2-keto-D-gluconic acid (PDB ID: 5v7n), and an enzyme from Aquifex aeolicus that belongs to the X9 subfamily (O66939_AQUAE) in complex with a cofactor and unknown ligand, interpreted as a complex with NADH and lactate (PDB ID: 3 kb6) [41] (Fig. 4). As seen in Fig. 4, one of the ligands (4-methylthio-2-oxobutyric acid bound to CtBP1 in complex with $\mathrm{NAD}^{+}$; PDB ID: 4lce) is modeled in a slightly unusual orientation, with the carboxyl group rotated around the $\mathrm{C} 1-\mathrm{C} 2$ bond. This ligand corresponds to the unusual $\mathrm{C}^{2}$ '-endo conformation of the ribose moiety of the cofactor seen in the same figure. However, both the ligand and the cofactor show incomplete occupancy and poor electron density in the crystal structure and should be interpreted with caution. To address this issue, we downloaded the structural model and structure factors of 4lce that were deposited in the PDB and re-refined this structure using recently published guidelines [42]. Re-refinement revealed that the sugar moiety is likely in the $\mathrm{C} 3$ '-endo conformation (as in all other $2 \mathrm{HADH}$ structures) and that the orientation of the keto-acid is largely consistent with other structures.

These structures show the mode of 2-keto/2-hydroxy acid binding by $2 \mathrm{HADH}$ that involves four highly conserved residues (Gly73, Arg230, Glu259, and His277, conserved in more than $90 \%$ of all $2 \mathrm{HADH}$ sequences) and a variable residue that bind the substrate via a main-chain amide (Val72). Two consecutive main chain amines from the $\beta 4-\alpha 4$ loop (Fig. 3, Fig. 4) form hydrogen bonds to the carboxylate atoms of the substrate, positioning the carboxyl group and thus orienting the substrate relative to the cofactor. The first amide comes from Val72, which is often replaced with another small residue such as alanine, serine, or threonine (Additional file 7: Figure S3). The second amide comes from a highly conserved glycine (Gly73). The only exception from this general pattern is subfamily $\mathrm{X} 13$, which has asparagine and leucine residues in these consecutive positions, respectively. Arg230 is the most conserved residue in the active site because only arginine provides the positively charged guanidinium group that can bind the substrate via two atoms and thus properly orient the substrate in addition to stabilizing its charge. The guanidinium group binds both the reducible/oxidizable keto/hydroxyl oxygen, presumably leading to its polarization, and the single oxygen of the substrate carboxylate, thus contributing to the orientation of the substrate [38]. His277 is
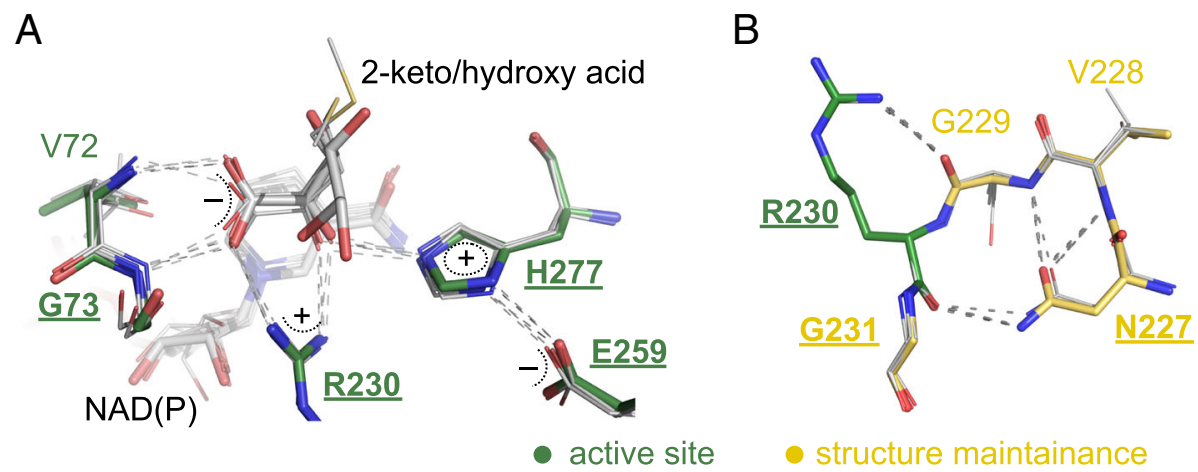

Fig. 4 Active site of canonical 2HADHs: (a), active site residues, reaction substrates/products (2-keto acids/2-hydroxy acids), and cofactors $[\operatorname{NADP}(H)$ or NAD $(H))] ;(\mathbf{b})$, structural support of the active site arginine. Shown are selected residues of five ternary complexes: $S$. meliloti GHRB with 2-keto-D-gluconic acid and NADP ${ }^{+}$(PDB ID: 5V7n, shown in wider sticks), human GRHPR with 2,3-dihydroxypropanoic acid and NADP ${ }^{+}$(PDB ID: 2gcg), human CTBP1 with 4-methylthio-2-oxobutyric acid and NAD (PDB ID: 4lce), human CTBP2 and 4-methylthio-2-oxobutyric acid and $\mathrm{NAD}^{+}$(PDB ID: 4lcj), and A. aeolicus subfamily $X 9$ member with lactic acid and NAD (PDB ID: 3 kb6). Oxygen and nitrogen atoms are shown in blue and red, respectively, with carbon atoms in green (for PDB ID: 5V7n) or gray (in other structures). Hydrogen bonds between protein residues and product are shown with gray dashed lines. Residues are labeled according to the sequence of PDB ID: 5V7n. Labels of highly conserved residues (i.e., present in $>90 \%$ of $2 \mathrm{HADH}$ sequences) are shown in bold and underlined 
involved both in substrate binding and a "proton shuttle" system between the histidine and the carboxylic acid residue Glu259. Notably, the four residues responsible for direct binding of the core of the substrate belong to both the catalytic (Val72 and Gly73) and the cofactor-binding domains (Arg230 and His277), thus making the interdomain cleft closure a necessary prerequisite for catalysis. The roles of the active site residues in the catalysis are supported by multiple mutagenesis studies [21, 43-46].

The residues Arg230, Glu259, and His277, often referred to as the "catalytic triad" [39] are conserved in almost all 2HADHs (Fig. 3). However, there are single cases of substitutions in these positions. In most FDHs, glutamate is substituted with glutamine, which broadens the optimal $\mathrm{pH}$ range for substrate binding [46]. Histidine, which is thought to exchange a proton in the redox reaction, is substituted by lysine in SERA type IIIK, and by tryptophan in X4 (PDB ID: 4njo and 1qp8, respectively; Additional file 8: Figure S4). Despite the histidine substitution for lysine and the absence of glutamate, a SERA type IIIK enzyme was shown to be catalytically active, presumably because lysine is also capable of maintaining two protonated states of the side chain [47]. However, the indole nitrogen in tryptophan is never protonated under physiological conditions $\left(\mathrm{p} K_{\mathrm{a}}=-2.4\right)$ and it cannot lose the proton; thus, it cannot serve as a catalytic residue that would provide a proton for the catalysis. The molecular function of the members of X4 with tryptophan instead of histidine should still be studied experimentally (see section: New uncharacterized subfamilies).

\section{Residues maintaining the structure}

Among the three conserved residues with structural function, asparagine (Asn227) and glycine (Gly231), located in the $\beta E-\alpha G$ loop, are responsible for positioning and conformational stabilization of the catalytic arginine Arg230 (Fig. 4). Gly231, which follows Arg230, gives the conformational flexibility to the protein main chain that is necessary to position the arginine side chain in the proper orientation. Asn227, separated by two residues from the arginine, locks the arginine main chain by forming hydrogen bonds to its main chain oxygen and to the main chain nitrogen of Gly229. Asn227 is highly conserved in $2 \mathrm{HADHs}$, because asparagine side chain has the capacity of forming the two hydrogen bonds with the main chain atoms (one atom accepts hydrogen, and the other is donor, Fig. 4). This highly specific conformation of the polypeptide chain requires extra conformational flexibility of the main chain around the residue preceding Arg230, which is provided by glycine (Gly229). Interestingly, Gly229 is not highly conserved in 2HADHs and is often replaced by a residue with a small side chain (Ala, Ser). However, these residues are always found to be Ramachandran plot outliers in all known crystal structures (PDB IDs: 5tx7, 5dt9, 3oet, 2o4c, and others).

The third residue, located downstream of the crossover helix $\alpha \mathrm{E}$ of the cofactor-binding domain, usually aspartate (93\% of the sequences; Asp195), is substituted to arginine in $3 \%$ of the sequences. This residue forms hydrogen bonds to residues in adjacent loops, probably contributing to the maintenance of the fold.

\section{Residues contributing to substrate specificity}

Based on the collected data, an enzyme with narrow substrate specificity in the $2 \mathrm{HADH}$ family is exceptional, and different subfamilies, separated early in evolution, often exhibit similar substrate profiles (Fig. 1, Additional file 4: Table S1). Therefore, determination of positions crucial for substrate specificity (which we term "specificity determining positions") that are general for the entire family is a particularly difficult or, maybe even impossible, task. Previous analyses of solved crystal structures and sequence alignments suggested that specific residues govern substrate discrimination within single subfamilies [29, 35, 38, 39]. In addition, some attempts were made to change substrate specificity of single enzymes by introducing point mutations in the proximity of the active site [48]. However, the mutagenesis data is scarce and hypotheses about molecular features governing substrate specificity among $2 \mathrm{HADHs}$ are largely based on crystal structures of ternary complexes solved with inactive substrate analogs or products.

Substrate specificity stems largely from the acquisition of unique loop regions and the adaptation of the physico-chemical nature of the substrate-binding pocket. Our analysis of available crystal structures shows that residues that can contact a variable substituent at the $\mathrm{C} 2$ carbon atom are found-depending on the structure and substrate-in up to eight regions of the sequence (Fig. 3, Additional file 8: Figure S4). Four of them are supplied by the substrate-binding domain (loops $\beta 1-\alpha 1, \beta 3-\alpha 3$, $\beta 4-\alpha 4$, and helix $\alpha 5$ ). Two regions are located within loops connecting the two domains (loops $\beta 5-\alpha \mathrm{A}$ and $\beta G-\alpha 5)$. Selectivity in $2 \mathrm{HADH}$ also appears to be dependent on the interactions within oligomeric assemblies: in many complexes, some residues in the substrate pocket, usually aromatic or acidic, are supplied by another subunit of a dimer (i.e., $\alpha B^{\prime}-\beta A^{\prime}$ ). Furthermore, in PDXB, a dimerization domain specific to PDXB can supplement the pocket with a substrate-binding arginine residue (PDB ID: 2o4c, "PDXB_dim" in Additional file 7: Figure S3).

Due to local structural changes of the enzymes, the broad range of physicochemical properties of their substrates, and the location of the catalytic pocket at the interface of two domains, 2HADH subfamilies developed various modes of substrate discrimination (Additional 
file 8: Figure S4). Below, we provide the first attempt to systematically characterize the contributions of specific regions of $2 \mathrm{HADH}$ structures to the substrate specificity of subfamilies, based on available crystal structures. However, as noted before for other enzyme families, substrate specificity may go beyond the physicochemical and steric characteristics of the active site, i.e., it may depend on global protein dynamics, the transition from the 'close' to 'open' conformation, and the mechanism of substrate entrance/exit $[49,50]$.

\section{Descriptions of the subfamilies}

The properties of the nine biochemically studied subfamilies (Fig. 1) are summarized in Table 1. Figure 3 shows the sequence logos of their regions critical for substrate and cofactor binding. Figure 5 shows their abundance in the genomes of model organisms.

\section{CTBP}

C-terminal binding proteins (CtBPs) can be found in vertebrates (e.g., rats and humans) and arthropods (e.g., Drosophila melanogaster [51]), yet members studied enzymatically include only two human paralogs. The animal CtBPs localize to both the nucleus and the cytoplasm, and much effort has been made to study transrepression pathways in which they may be involved [52].

CtBPs were first identified as transcriptional corepressors targeting many transcriptional regulators [53] and playing critical roles during development of both invertebrates and vertebrates [52]. Although the precise mechanism of the corepressor activity is still under investigation, it is known that CtBPs recognize the consensus PXDLS motif in DNA-binding and other transcription-related proteins $[54,55]$. Later studies confirmed that they also possess dehydrogenase activity, and the $\mathrm{NAD}^{+}$-dependent conformational change is thought to be essential to their corepression activity [56, 57]. Human CtBP1 (CTBP1_HUMAN) reduces a number of substrates, including glyoxylate and pyruvate, with relatively low activity, using NADH as a cofactor [58] ( $\beta \mathrm{B}-\alpha \mathrm{D}$ loop, Fig. 3). Human CtBP1 shows the highest catalytic efficiency with 4-methylthio-2-oxobutyric acid (MTOB), an intermediate of the methionine salvage pathway [58]. The saturation curve shows biphasic behavior, with marked substrate inhibition at elevated concentrations [58]. Nevertheless, the most relevant physiological substrates for CtBPs and their role in the corepressor function remain unclear.

The homolog from A. thaliana (CTBP_ARATH, C-terminal binding protein AN) differs substantially from the animal CtBPs in sequence, lacks the catalytic residues, and does not seem to regulate transcription [59]. For this reason, the plant homologs were not included in the CTBP subfamily and considered as its sister clade.
Based on the available crystal structures solved in complex with a cofactor and MTOB, it was observed that the sulfur atom of MTOB forms a sulfur- $\pi$ interaction with tryptophan from the $\beta$ G- $\alpha 5$ loop (Trp318 in PDB ID: 4lce, Additional file 8: Figure S4). This interaction is thought to confer specificity towards MTOB [40]. Other bulky residues, such as a conserved histidine and tyrosine from the $\beta \mathrm{G}-\alpha 5$ loop, form the tight binding pocket and appear to constrain the size of substrates accepted.

In the case of CtBP1, the tetramer assembly is preceded by a dimeric intermediate, in which the tryptophan (Trp318) from the $\beta G-\alpha 5$ loop functions as a switch for effective dimerization following $\mathrm{NAD}^{+}$binding (Additional file 8: Figure S4, PDB ID: 4lce) [60, 61]. Mutation of this residue to phenylalanine reduced dimerization and completely abolished tetramerization, what suggested that $\mathrm{NAD}(\mathrm{H})$-dependent dimerization occurs with Trp318 required to effectively induce the strand switch, bringing the dimer pairs into a spatial context permissive for homotetramerization [61].

\section{DDH}

This subfamily is named after the only biochemically studied representative, D-2-hydroxyacid dehydrogenase (Ddh) from Haloferax mediterranei (DDH_HALMT), and comprises proteins spread over the taxonomic tree, including eukaryotes (e.g., fungi and protists), archaea and bacteria (cyanobacteria and actinobacteria). Ddh from H. mediterranei catalyzes the reduction of a broad range of 2-ketocarboxylic acids, with a preference for those having an unbranched chain of 4-5 carbon atoms, such as 2-ketoisoleucine [62]. It exhibits dual cofactor specificity, yet shows better catalytic efficiency with NADPH [62]. The sequence conservation within the $\beta \mathrm{B}-\alpha \mathrm{D}$ loop does not display the respective aspartate residue defining the specificity towards $\mathrm{NAD}(\mathrm{H})$ (Fig. 3), suggesting that most enzymes within the DDH subfamily would display preference towards NADPH. Although some archaeal genomes (e.g., the halophilic mesophile Haloferax volcanii, Fig. 5) encode as many as four DDH representatives, their function is not known.

Recently, three crystal structures of DDH_HALMT were solved in complex with combinations of $\mathrm{NAD}^{+}, \mathrm{NADP}^{+}$, NADPH, 2-ketohexanoic acid, and 2-hydroxyhexanoic acid (PDB IDs: 5mha, 5mh5, 5mh6). Although the DDH subfamily displays high sequence variability, some common features can be distinguished based on the sequence alignment with other 2HADH subfamilies (Fig. 3). For example, the $\beta 1-\alpha 1$ loop harbors a considerably long insertion, which folds into a tightly packed 3/10-helix in the crystal structures. Furthermore, a highly conserved tryptophan within the $\alpha B-A$ loop from the other subunit (Trp122 in DDH_HALMT), which is thought to preclude larger 


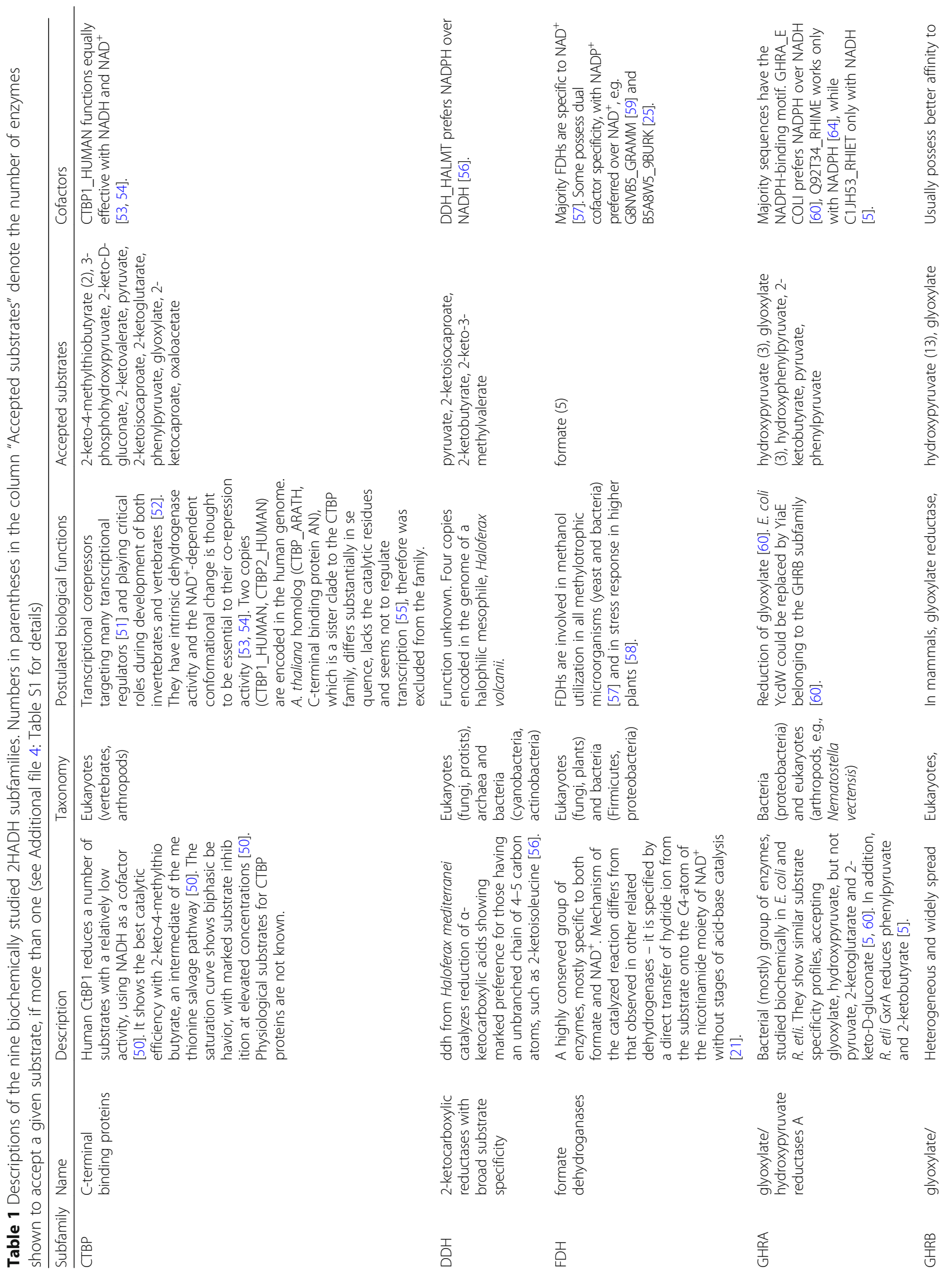




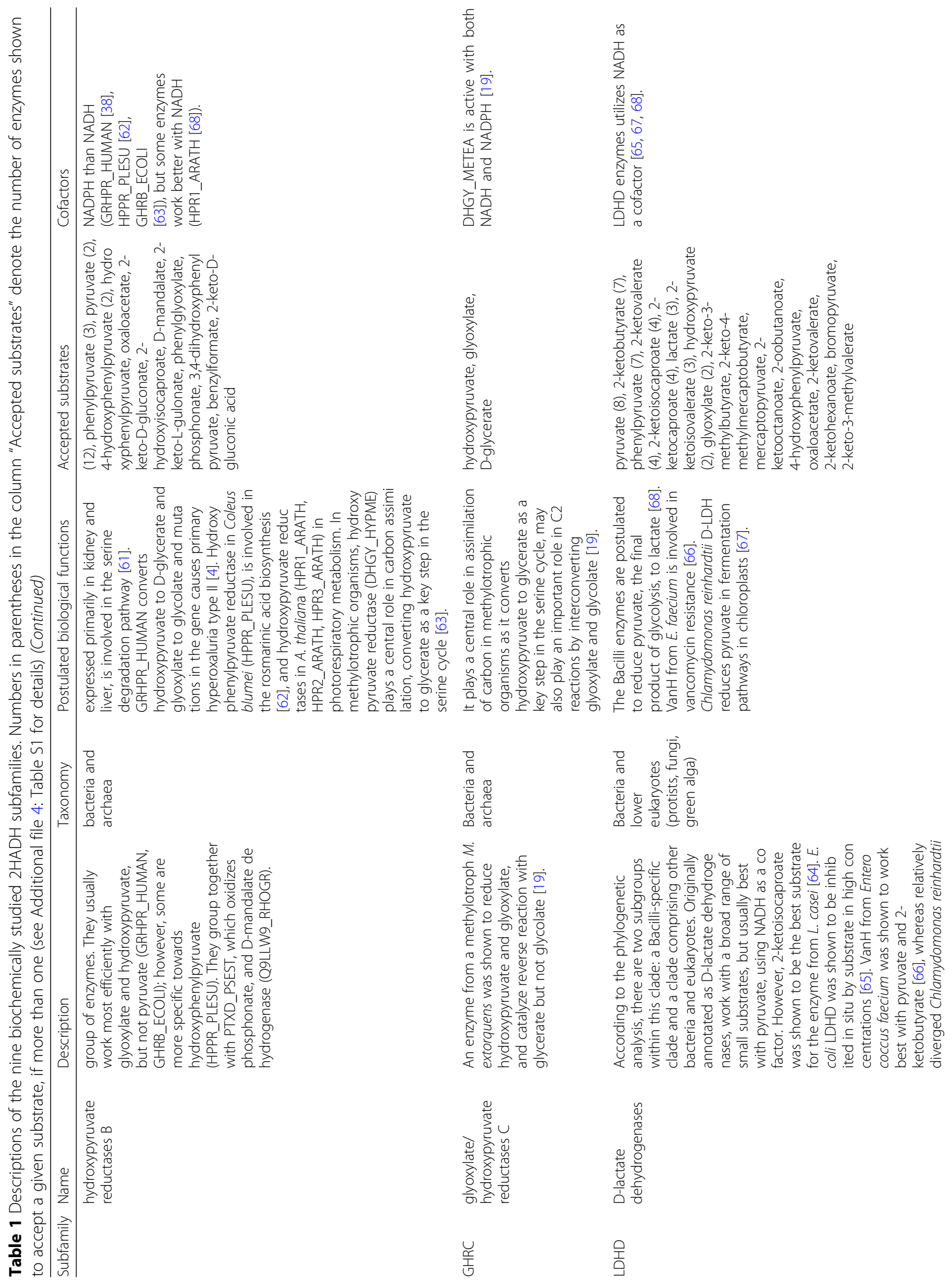




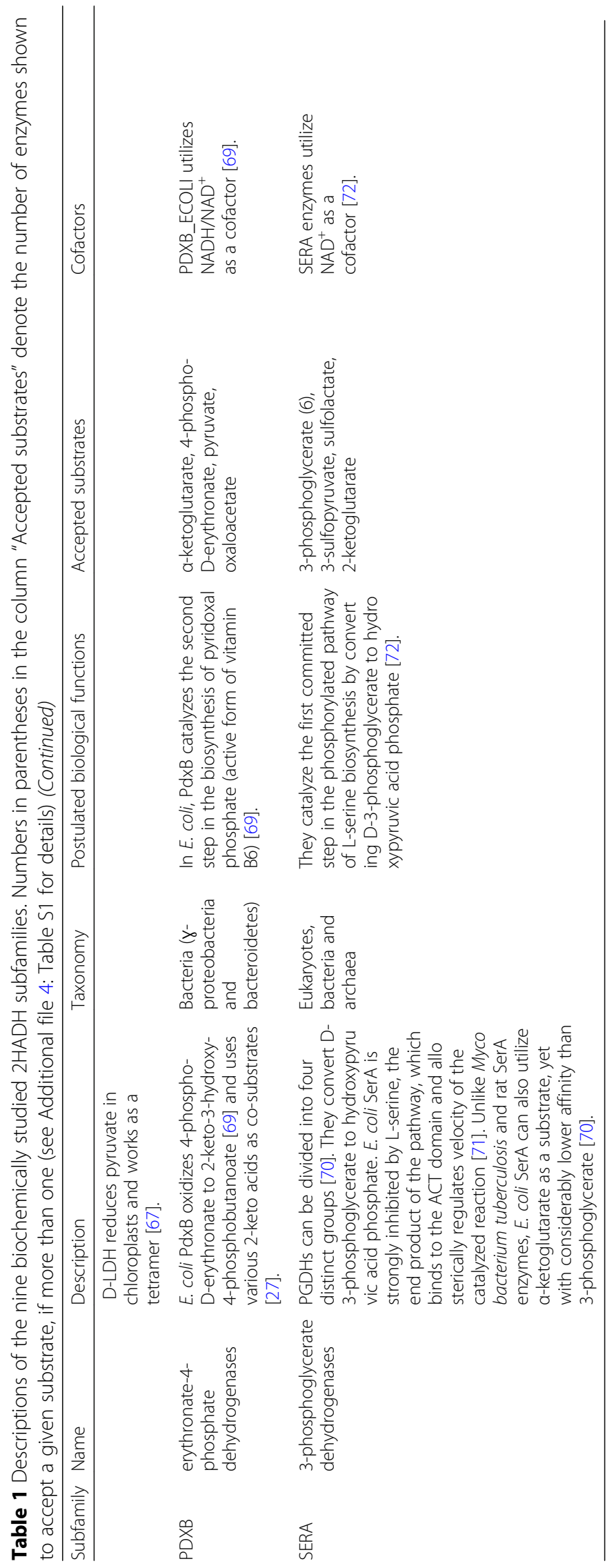



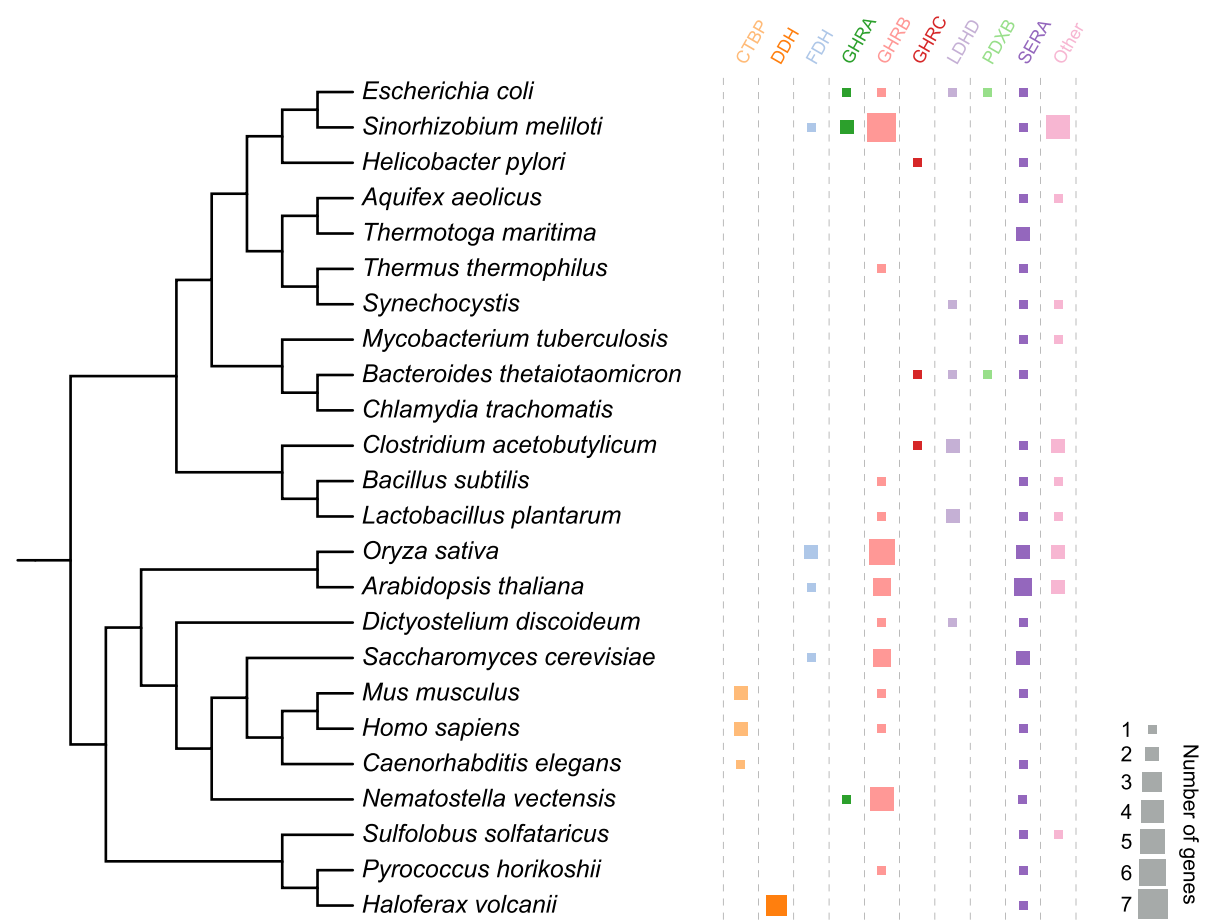

Fig. 5 Abundance of the nine biochemically studied 2HADH subfamilies in selected model organisms. The size of each square corresponds to the number of proteins belonging to a given subfamily encoded in the given organism. The tree topology was obtained from iTOL [112], and proteomes were downloaded from KEGG [113] (Additional file 9: Data file S2)

substrates from binding to members of the GHRA, GHRB, and SERA subfamilies, is located far away from the active site (Additional file 8: Figure S4). In addition, small residues within the $\beta 5-\alpha$ A loop would allow accommodation of large and hydrophobic substrates, whereas large residues facing the active site from the 3/10-helix (such as Arg14 in DDH_HALMT) could possibly prevent the binding of branched substituents (Additional file 8: Figure S4).

\section{FDH}

Formate dehydrogenases (FDHs) represent a highly conserved subfamily of enzymes, characterized by a scattered taxonomic distribution. They are present in various bacteria (i.e., Firmicutes and proteobacteria) and eukaryotes (plants, yeasts, and fungi), and catalyze the $\mathrm{NAD}^{+}$-dependent oxidation of formate to carbon dioxide. The aspartate residue that defines the specificity for $\mathrm{NAD}^{+}$is conserved within the subfamily $(\beta \mathrm{B}-\alpha \mathrm{D}$ loop, Fig. 3). The formate oxidation is the simplest reaction catalyzed by $2 \mathrm{HADHs}$, as it lacks the proton release step. It is specified by a direct transfer of hydride ion from the substrate onto the $\mathrm{C} 4$ atom of the nicotinamide moiety of $\mathrm{NAD}^{+}$. Since formate is not a 2-hydroxy acid, the mode of substrate binding in FDH differs from other 2HADHs [21]. FDHs constitute the most studied $2 \mathrm{HADH}$ subfamily, and their characteristics have been reviewed extensively $[33,63]$.
FDHs play a pivotal role in methanol utilization in methylotrophic microorganisms (yeast and bacteria), supplying them with energy and reducing equivalents [21]. As opposed to microbiological FDHs, which function in cytoplasm, plant FDHs localize to the mitochondria and are key players in the cell stress response caused by both exogenic and endogenic factors [33].

The vast majority of FDHs studied so far accept only formate as a substrate and $\mathrm{NAD}^{+}$as a cofactor, though some were found to possess double cofactor specificity $[25,64]$. A number of enzymes have also been shown to oxidize esters and thioesters of formic acids [63]; however, the physiological significance of the additional substrates has not been confirmed.

Interestingly, although FDHs are considered highly specific enzymes, they possess relatively low affinity to formate, characterized by a $K_{\mathrm{M}}$ of $3-10 \mathrm{mM}$ [21]. Affinities to the cofactor are usually 1-2 orders of magnitude higher, with most $K_{\mathrm{M}}$ values ranging from 10 to $100 \mu \mathrm{M}$ $[27,65,66]$. Similarly, catalytic efficiencies are relatively small, yet higher in bacterial FDHs than methylotrophic yeast FDHs. Improvement of the catalytic parameters of FDHs by genetic engineering is an important issue, as FDH enzymes are widely used for NADH regeneration in enzymatic syntheses of optically active compounds [21].

A wealth of structural data [21, 30, 35] and computational studies $[67,68]$ is available for the FDH subfamily, 
making it one of the most studied 2HADH subfamilies. As reflected by the high sequence similarity among its members (Fig. 1), their active site environment is almost invariable. A common feature of all FDHs is an extended $\beta 3-\alpha 3$ loop harboring the $\mathrm{PF}[\mathrm{HW}] \mathrm{P}$ sequence motif, which appears to significantly reduce the size of the active site. The invariant prolines maintain the aromatic residues in stereochemically constrained positions, further supported by stacking of their aromatic rings (Additional file 8: Figure S4). In consequence, the active site remains rigid and tightly packed, perfectly tailored for accommodation of small substrates.

\section{GHRA}

In previous classifications $[5,6]$, this subfamily (represented by Rhizobium etli GxrA and E. coli GhrA) was classified jointly with GHRB. However, in our evolutionary trees, it consistently appeared as polyphyletic with GHRB and clustered closely with DDH. Also, even in the phylogenetic trees underlying the previous classification, GHRA emerged and separated early from GHRB $[5,6]$. This subfamily comprises mostly bacterial enzymes (except for one protein from Nematostella vectensis, closely related to $\beta$-proteobacterial enzymes), of which three (from E. coli, R. etli, and S. meliloti) have been characterized biochemically [5, 69]. Unlike most GHRB members, which have hydroxypyruvate as a preferred substrate, they have been shown to work most efficiently towards glyoxylate. Their secondary substrates include hydroxypyruvate, phenylpyruvate and pyruvate, but not 2-keto-D-gluconate, which is a substrate for several GHRB members. In our recent study [70], we highlight differences between the GHRA and GHRB clades by structural and enzymatic characterization of two members from S. meliloti 1021.

Enzymes that belong to the GHRA clade exhibit high sequence similarity. They share a conserved sequence fingerprint for specificity towards NADPH at the pocket shown to bind adenine and ribose moieties of the cofactor $([\mathrm{ST}] \mathrm{R}[\mathrm{ST}] \mathrm{X}[\mathrm{RK}]$ in the $\beta \mathrm{B}-\alpha \mathrm{D}$ loop, Fig. 3) [71]. In vitro, E. coli and $S$. meliloti representatives were indeed shown to be selective for NADPH over NADH. However, R. etli GxrA was reported to work only with NADH [5], which seems dubious, because the sequence of $R$. etli GxrA has the fingerprint of specificity for $\mathrm{NADPH}$. The physiological function of the enzymes remains to be discovered. The E. coli GhrA was proposed to contribute to glyoxylate reduction in the cell, yet in a dispensable manner [69].

Crystal structures of the GHRA homologs bound with substrate analogs revealed a large hydrophobic active site with a conserved tryptophan from the $\beta 3-\alpha 3$ loop (Trp53 in R. etli, S. meliloti or Xanthobacter autotrophicus GhrA, PDB IDs: 5tsd, 4z0p or 5vg6, respectively) interacting with $\mathrm{C} 2$ atom substituents. The tryptophan is unique to the GHRA subfamily and probably contributes to selection for smaller hydrophobic or aromatic substrates [70].

\section{GHRB}

This subfamily is characterized by the broadest substrate selectivity and highest diversity in function among 2HADHs. Members of the GHRB clade bind a large variety of putative physiological substrates, as diverse as glyoxylate, hydroxypyruvate, phosphonate, D-mandalate, 2-keto-D-gluconate, phenylpyruvate, and 3,4-dihydroxyphenylpyruvate (Table 1). At the same time, they exhibit a high level of promiscuity, i.e., they accept various secondary substrates, which occasionally appear as the most efficient substrates for the most similar homologs. In terms of $k_{\text {cat }} / K_{\mathrm{M}}$ values, they are less active than LDHDs, with maximal values of over $10^{5} \mathrm{M}^{-1} \mathrm{~s}^{-1}$, and although they have similar substrate profiles, GHRBs usually do not accept pyruvate. Enzymes falling into this subfamily typically possess better affinity for NADPH than for NADH (e.g., GRHPR_HUMAN, HPPR_PLESU, and GHRB_ECOLI), but individual proteins are shown to work more efficiently with NADH (e.g., HPR1_ARATH).

This heterogeneous subfamily spans enzymes from all kingdoms of life (Fig. 5). Among its representatives are yeast mandalate dehydrogenase [24], human and archaeal glyoxylate/hydroxypyruvate reductases [4, 72], bacterial phosphonate dehydrogenases [23], plant and fungal hydroxyphenylpyruvate reductases $[6,73]$, and bacterial enzymes reducing broad ranges of substrates $[5,69]$.

As hydroxypyruvate and glyoxylate constitute important compounds in various metabolic pathways, GHRB members play crucial roles in many biological processes. Mammalian glyoxylate reductase has a potentially protective role by metabolizing glyoxylate to the less reactive glycolate [4]. Hydroxyphenylpyruvate reductase from Coleus blumei (HPPR_PLESU) is involved in rosmarinic acid biosynthesis [73], while hydroxypyruvate reductases from A. thaliana (HPR1_ARATH, HPR2_ARATH, and HPR3_ARATH) are involved in photorespiratory metabolism [74]. In methylotrophic organisms, hydroxypyruvate reductase (DHGY_HYPME) plays a central role in carbon assimilation, converting hydroxypyruvate to glycerate as an essential step in the serine cycle [75]. 2-keto-D-gluconate dehydrogenase from Gluconobacter oxydans (2KGR_GLUOX) is responsible for the utilization of the compound as a carbon source [76].

The recent age of the duplications and evidence of a horizontal gene transfer in the recent history of the GHRB subfamily suggest that the function and enzymatic behavior of its members could be extrapolated to a limited extent. Surprisingly, some genomes encode as 
many as six GHRB paralogs (Fig. 5). In the $\mathrm{N}_{2}$-fixing a-proteobacterium Sinorhizobium sp. NGR234, a majority of the GHRB homologs are expressed at relatively low levels (i.e., less than 100 reads per kilobase per million mapped reads, RPKM) [77]. As shown for PprA from Wickerhamia fluorescens TK1, their transcription could adapt to some specific metabolic conditions [6].

The enzymatic diversity of GHRB is reflected by the active site environments in the known crystal structures. Presence of the Gly-Ser motif within the $\beta G-\alpha 5$ loop is correlated with the highest activity with hydroxypyruvate (e.g., Pyrococcus horikoshii GYAR_PYRHO, H. sapiens GRHPR_HUMAN, Plectranthus scutellarioides HPPR_PLESU, and S. meliloti Q92LZ4_RHIME). The serine side chain (e.g, Ser296 in PDB ID: 2gcg and Ser280 in PDB ID: 5v7n, Additional file 8: Figure S4) is thought to be responsible for discrimination for hydroxypyruvate due to the formation of a hydrogen bond with its hydroxyl group [70]. Another highly conserved motif - Arg-X-X-Met - is located within the a5 loop in most GHRB members. Probably the large side chains of the Arg and Met residues prevent the binding of substrates containing larger $\mathrm{C} 2$ substituents that extend the C1-C2 plane (e.g., PDB ID: 5v7n, Additional file 8: Figure S4). In Rhodotorula graminis Q7LLW9_RHOGR, the motif corresponds to Phe-His-Glu-Phe and correlates with high activity of the enzyme towards D-mandalate. Another important residue contributes from the other subunit of the dimer (Trp141 in PDB ID: 2gcg, Trp134 in PDB ID: 4e5k, Additional file 8: Figure S4). This large aromatic residue potentially precludes the binding of larger substrates: its absence in S. meliloti Q92LZ4_RHIME coincides with activity for larger substrates, such as 2-keto-D-gluconate [70]. Variable residues within the $\beta 3-\alpha 3$ loop may be also involved in distinguishing physicochemical properties of the substrate. Large hydrophobic side chains, such as Leu59 in GRHPR_HUMAN or Leu70 in HPR1_ARATH, might prevent binding of substrates with large substituents, whereas small hydrophilic residues, such as Ser50 in Q92LZ4_RHIME, might promote selection towards large hydrophilic substrates.

\section{PTXD}

In a majority of the reconstructed phylogenetic trees, the GHRB subfamily contains a small clade, PTXD, containing (among others) phosphonate dehydrogenase from Pseudomonas stutzeri (PTXD_PSEST). The enzyme catalyzes the oxidation of phosphite to phosphate coupled to the stoichiometric reduction of $\mathrm{NAD}^{+}$to $\mathrm{NADH}$; besides, it was shown to reduce hydroxypyruvate at a low level [23]. None of other tested compounds were reduced by the enzyme; however, it has not been tested against glyoxylate and phenylpyruvate, which are common substrates for GHRB members.
Several structures of P. stutzeri PtxD variants with improved thermostability and catalytic efficiency have been solved (Additional file 8: Figure S4, PDB ID: 4e5k) [78, 79]. It was suggested that highly hydrophobic residues that interact with the substrate analog (Met53, Leu75, and Leu100 in PTXD_PSEST) contribute to closing off the active site [79]. The tight substrate-binding pocket is shielded by Trp314 provided by the other subunit of the dimer. However, mutagenesis studies indicate that the tryptophan is not important for catalysis [80]. Another residue from the active site, Arg301 located within helix a5, is thought to contribute to electrostatic interactions with negatively charged substrates [80]. It is fully conserved in PTXD homologs, but not in other 2HADH enzymes. Interestingly, the R301K mutant displayed a slightly higher $k_{\text {cat }}$ than the parent PTXD, and a more modest increase in $K_{M}$ for phosphite [80]. Although three other residues-Trp314 (mentioned above), Tyr139, and Ser295-are specific for PTXD orthologs, site-directed mutagenesis proved them not important for the catalysis [80].

\section{GHRC}

In addition to GHRA, GHRB, and DDH, another clade of bacterial and archaeal proteins, here termed GHRC, emerged to include a glyoxylate/hydroxypyruvate reductase. The only biochemically characterized member of the subfamily-an enzyme from a methylotroph Methylobacterium extorquens (DHGY_METEA)-was shown to reduce hydroxypyruvate and glyoxylate, and to catalyze the reverse reaction with glycerate [19]. It was proposed to play a central role in the assimilation of carbon in methylotrophs, as it converts hydroxypyruvate to glycerate (a key step in the serine cycle) [19]. The enzyme was shown to utilize both NADH and NADPH as a cofactor. However, it is not known to what extent the characteristics of this enzyme apply to other members of this subfamily. The region responsible for cofactor specificity (i.e., the $\beta B-\alpha D$ loop) does not contain the characteristic aspartate residue that defines the preference for $\mathrm{NADH}$, suggesting that NADPH may be the preferred cofactor (Fig. 3).

A crystal structure of a GHRC representative from Desulfovibrio vulgaris has been solved (PDB ID: 5tx7). Although the structure is in apo form, arrangement of the domains suggests that it adopts a closed conformation. The tight substrate pocket is lined with two tryptophan residues (Trp135 and Trp288), absolutely conserved in the GHRC subfamily, and a lysine residue (Lys52, Additional file 8: Figure S4). The lysine is located within the "Asn-Lys" motif at the $\beta 3-\alpha 3$ loop and is present in almost all GHRC sequences. The large polar environment created by large aromatic residues is rarely seen in other subfamilies (Additional file 8: Figure S4), 
and may be used for other small and hydrophilic substrates not tested in the previous biochemical assay.

\section{LDHD}

Enzymes from this subfamily can be found in bacteria and some lower eukaryotes, such as protists, fungi and green algae. Bacterial proteins initially annotated as D-lactate dehydrogenases (LDHD, or D-LDH) act at the last step of glycolysis in anaerobic conditions, by catalyzing the reduction of pyruvate to D-lactate, allowing regeneration of $\mathrm{NAD}^{+}$from $\mathrm{NADH}$ [81]. These enzymes may also play a role in other processes, as demonstrated for VanH, which is responsible for vancomycin resistance in Enterococcus faecium [82]. The only eukaryotic D-LDH studied so far, the Chlamydomonas reinhardtii enzyme, was shown to reduce pyruvate in fermentation pathways in chloroplasts [83]. According to the proposed role of $\mathrm{NAD}^{+}$regeneration, this subfamily has the highly conserved characteristic aspartate residue that defines the preference towards $\mathrm{NADH}$ in the the $\beta \mathrm{B}-\alpha \mathrm{D}$ loop (Fig. 3).

According to our phylogenetic analysis, there are two subgroups within this subfamily: a Bacilli-specific clade (e.g., LDHD_LACPL) and another one comprising other bacteria (e.g., LDHD_ECOLI) and eukaryotes (BOLUZ5_ CHLRE, Fig. 1). LDHD members usually exhibit the highest catalytic efficiency towards pyruvate (with $k_{\text {cat }} / K_{\mathrm{M}}$ over $10^{6}$ $\mathrm{M}^{-1} \mathrm{~s}^{-1}$ ) and were also shown to accept other small compounds, such as glyoxylate and 2-ketobutyrate, with considerably lower efficiency. One exception is D-2hydroxyisocaproate dehydrogenase $(R-\mathrm{HicDH})$ from Lactobacillus casei (Q03CR3_LACC3, DHD2_LACPA), which clusters closely with typical Bacilli D-lactate dehydrogenases. In a systematic screening, $R$-HicDH was shown to reduce a broad range of substrates, including straight and branched aliphatic 2-keto acids, with phenylpyruvate and 2-ketoisocaproate with the highest $k_{\mathrm{cat}} / K_{\mathrm{M}}$ and $K_{\mathrm{M}}$, respectively [84]. The $k_{\text {cat }} / K_{\mathrm{M}}$ value was three orders of magnitude lower for pyruvate. $R$-HicDH slowly catalyzes reactions with medium-size carboxylates, which, unusually, do not follow conventional Michaelis-Menten kinetics, possibly due to weak substrate binding [84]. In addition, E. coli LDHD was shown to be inhibited in situ by the substrate in high concentrations [85].

Analyses of crystal structures describe the architectures used by LDHDs to control the size and electrostatic character of the substrate-binding site [39]. Attention was especially brought to residues from loops $\beta 3-\alpha 3, \beta 5-\alpha A$ and $\beta G-\alpha 5$ [39]. The residues at $\beta 3-\alpha 3$ play a steric role in substrate selectivity: hydrophobic and aromatic phenylalanine (e.g., Phe51 in PDB ID: $3 w \times 0$, LDHD_ECOLI) is thought to prevent binding of substrates larger than pyruvate, whereas smaller leucine (e.g., Leu51 in PDB ID: 1dxy, DHD2_LACPA) and glycine (Gly54 in VANH_ENTFC) contribute towards the broader substrate specificity of LDHDs $[28,29]$. The amino acids at the $\beta 5-\alpha \mathrm{A}$ and $\beta$ G- $\alpha 5$ loops appear to be conserved within the LDHD subfamily: tyrosine and phenylalanine/tyrosine, respectively, presumably restrict the space for $\mathrm{C} 2$ substituents [28]. Interestingly, Arg9 from the $\beta 1-\alpha 1$ loop in $R$-HicDH from Lactobacillus paracasei (PDB ID: 1dxy) was proposed to be responsible for the non-Michaelis-Menten kinetics observed for this enzyme. Because of its proximity to the active site, Arg9 may compete with the arginine of the catalytic triad for the substrate and lead to non-productive substrate binding (Additional file 8: Figure S4) [28]. However, this hypothesis awaits confirmation by site-directed mutagenesis studies.

\section{PDXB}

This small subfamily includes a group of bacterial enzymes found in $y$-proteobacteria and Bacteroidetes, including biochemically studied PdxB from E. coli (PDXB_ECOLI). PdxB oxidizes 4-phospho-D-erythronate to 2-keto-3-hydroxy-4phosphobutanoate and uses various 2-keto acids as cosubstrates, utilizing $\mathrm{NAD}^{+}$as a cofactor $[27,66]$. The reaction is the second step in the biosynthesis of pyridoxal phosphate - the active form of vitamin B6 [66]. The PDXB subfamily has a highly conserved characteristic aspartate residue that defines the preference towards NADH in the $\beta B-\alpha D$ loop (Fig. 3). Uniquely for $2 \mathrm{HADHs}$, PDXB family proteins have two consecutive proline residues within the loop, which are spatially conserved in all the crystal structures of PDXB proteins. Another unique feature of PDXB is presence of a C-terminal dimerization domain (Additional file 7: Figure S3).

PDXB contains three members (from Pseudomonas aeruginosa, Salmonella typhimurium, and Vibrio cholerae) with crystal structures of the holoenzymes (PDB IDs: $204 \mathrm{c}$, 3oet, and $5 \mathrm{dt} 9$ respectively). The $P$. aeruginosa $\mathrm{PdxB}$ structure has been solved with a substrate analog (tartaric acid) bound in the active site. Based on the structure, it was proposed that two conserved arginines and a tyrosine residue anchor the phosphate moiety of the native substrate via charge compensation and hydrogen bonds [34]. Arg44 is located within the Arg-Ser motif at the $\beta 3-\alpha 3$ loop, whereas Arg346 is located in the dimerization domain unique to PDXB (PDB ID: 2o4c, Additional file 8: Figure S4). Thus, the dimerization domain likely also plays a major role in substrate recognition. The absolutely conserved residue Tyr258 is located within the $\beta$ G- $\alpha 5$ loop at the junction between the cofactor-binding and substrate-binding domains and presumably contributes to precise positioning of the phosphate group within the active site. Notably, the proposed mode of binding vastly differs from the consensus model presented on Fig. 4. 


\section{Sera}

3-phospho-D-glycerate dehydrogenases (PGDH, or SERA) constitute the most widespread subfamily within $2 \mathrm{HADHs}$, present in almost all living organisms. They are involved in the first step of the phosphorylated pathway of L-serine biosynthesis from 3-phosphoglycerate, an intermediate of glycolysis [86]. They reversibly oxidize D-3-phosphoglycerate to hydroxypyruvic acid phosphate utilizing $\mathrm{NAD}^{+}$as a cofactor [86], with the respective aspartate residue defining the specificity for $\mathrm{NAD}(\mathrm{H})$ is highly conserved within the subfamily $(\beta B-\alpha \mathrm{D}$ loop, Fig. 3). According to the published biochemical studies, SERA members rarely catalyze other reactions.

SerA homologs were previously divided into four distinct groups - referred to as types I, II, IIIK, and IIIH $[86,87]$. Although the division was based mainly on the presence of additional regulatory domains, it is also reflected by the topology of the phylogenetic tree, computed based on the alignment of the cofactor-binding and substrate-binding domains (Fig. 1).

Type I enzymes are represented by the human, $M$. tuberculosis, and A. thaliana proteins. They act as tetramers and share a conserved domain architecture, where substrate-binding and cofactor-binding domains are followed by two regulatory domains-an allosteric substrate-binding ("ASB") domain and a regulatory motif recurring in many enzymes, termed aspartate kinasechorismate mutase-TyrA ("ACT") domain. The ACT region binds amino acids (in this case, L-serine) and functions in feedback inhibition of amino acid synthesis pathways [88]. As shown for M. tuberculosis PGDH, the second layer of regulation is provided by the ASB domain. It appears to modulate sensitivity to L-serine by phosphate and polyphosphate, which triggers a conversion between oligomers with different serine-sensitive states [89].

Type II PGDHs, comprising E. coli and yeast enzymes, contain only an additional ACT domain and also act as tetramers. As with type I enzymes, E. coli SerA is strongly inhibited by L-serine, which binds to the ACT domain and allosterically regulates the velocity of the catalyzed reaction [90]. It shows an exceptionally high affinity to $\mathrm{NADH}$, estimated as $50 \mathrm{nM}$ [91]. Unlike $M$. tuberculosis and rat SerA, the E. coli enzyme can also utilize $\alpha$-ketoglutarate as a substrate, yet with considerably lower affinity than 3-phosphoglycerate [87, 92].

Types IIIK and IIIH indicate type III dehydrogenases, which do not contain additional regulatory domains, with either lysine or histidine in the active site, respectively. Type III enzymes function as dimers, as opposed to type I and II, which are active as tetramers [86]. Type IIIK proteins are present in Bacteroidetes and protists, including Entamoeba histolytica [93]. According to crystal structures (PDB ID: 4njo) and mutagenesis studies
[47], the active site is formed by arginine and lysine residues (instead of the typical Arg/His/Glu triad). Lysine is thought to be an acid-base catalyst in the reaction, taking over the role of the catalytic histidine-glutamine pair. The type IIIH enzymes are present in bacteria and archaea, including proteins with a determined 3D structure from P. horikoshii (PDB ID: 1wwk) and Sulfolobus tokodaii (PDB ID: 2ekl).

In the reconstructed ML tree, 3-sulfolactate dehydrogenase SlcC from Chromohalobacter salexigens (SLCC_ CHRSD) is grouped within the SERA clade. However, this tree topology is not consistent among trees computed using alternative methodologies; plausibly, SLCC CHRSD could also be placed as a sister clade to SERA (Additional file 3: Data file S1). 3-sulfolactate is structurally similar to 3-phosphoglycerate, yet $\mathrm{SlcC}$ is involved in another pathway using 3-sulfolactate as a carbon source and does not accept 3-phosphoglycerate as a substrate [94].

Despite the relative sequence variability in the SERA subfamily, the available crystal structures show similar solutions for recognition of the negatively charged substrate, which is based on the presence of at least two conserved positively charged residues positioning the phosphate moiety (Additional file 8: Figure S4). In particular, the Arg-Ser motif located within the $\beta 3-\alpha 3$ loop, also present in the PDXB subfamily (Fig. 3), accommodates a SERA-invariant arginine that directly binds phosphate in the crystal structures. The arginine is usually stabilized by a conserved glutamine residue located in the $\alpha 5$ helix. The structural regions providing additional Arg/Lys residues depend on the SERA subtypes: In types $\mathrm{I}$ and $\mathrm{IIIH} / \mathrm{K}$, an arginine contacting the substrate is located in the $\beta 4-\alpha 4$ loop, whereas in all types except for IIIK, an extra Arg/Lys residue enters the active site from the other subunit within the $\alpha B^{\prime}-\beta A^{\prime}$ loop (Additional file 8: Figure S4).

\section{New uncharacterized subfamilies}

The 13 newly defined subfamilies that were not studied biochemically (X1-X13) constitute small clades, each comprising closely related species from bacteria, archaea, and plants (Fig. 1, Additional file 2: Figure S1). According to the sequence alignment, almost all new clades retain the conserved residues of the active site, thereby suggesting that these proteins probably act as active dehydrogenases or reductases (Additional file 7: Figure S3). Only the X13 subfamily lacks the conserved glycine residue (Gly73 in the $\beta 4-\alpha 4$ loop) that provides the amide atom to position the substrate relative to the cofactor, and $\sim 50 \%$ of members of the X4 subfamily have the histidine residue of the catalytic triad replaced with tryptophan, which is accompanied by a loss of the catalytic glutamate. 
Seven of the newly defined subfamilies include representatives with a determined 3D structure (Additional file 2: Figure S1). Among these structures, one was solved with a cofactor (PDB ID: 1qp8 from X4), another with a cofactor and a reaction product (PDB ID: $3 \mathrm{~kb} 6$ from X9, Additional file 8: Figure S4); the rest of the structures do not have function-relevant ligands (Additional file 6: Table S2). Below, we provide a short description of the two subfamilies that have at least one structure in complex with a cofactor.

The X4 subfamily consists of archaeal enzymes from Crenarchaeota and Euryarchaeota. The only structure in complex with a cofactor from this subfamily (PDB ID: 1qp8) corresponds to a protein from Pyrobaculum aerophilum PAE1175; it is annotated as a putative formate dehydrogenase in the PDB and as a 2-hydroxyacid dehydrogenase in UniProt. Notably, it lacks the catalytic His and Glu/Gln, instead containing Trp and Val in these positions, respectively. These substitutions are unique features of about $50 \%$ of the members of this subfamily (see section: Residues of the active site). Trp is conserved among PAE1175 homologs from Crenarchaeota, leading to questions regarding the molecular function of these enzymes. The substrate-binding domain of PAE1175 possesses a deteriorated version of the Rossmann fold, with a short hydrogen-bonded turn instead of $\alpha 2$ and a short $\beta$-bridge instead of $\beta 3$. Importantly, it lacks features characteristic of FDH subfamily members, such as a long $\beta 3-\alpha 3$ loop with the PF[HW]P sequence motif (Fig. 3) and Ile/Val within the $\beta 4-\alpha 4$ loop (Additional file 8: Figure S4). Therefore, as was concluded from an earlier analysis of its crystal structure, its role as a formate dehydrogenase seems highly farfetched [45]. However, since formate dehydrogenation is the only reaction catalyzed by $2 \mathrm{HADH}$ that does not involve proton transfer, it still may be the most feasible function of the Trp-substituted enzymes from the X4 subfamily. Interestingly, X4 members from Euryarchaeota, such as Ta0858 from Thermoplasma acidophilum with solved structure (PDB ID: 3gvx) possess the canonical Arg-Glu-His catalytic triad. According to the pattern of sequence conservation, most members of the subfamily likely display a preference for $\mathrm{NADP}(\mathrm{H})$ over NAD $(\mathrm{H})$, as the topology of the $\beta \mathrm{B}-\alpha \mathrm{D}$ loop is highly similar to that of GHRA (Fig. 3).

The only structure of an X9 subfamily protein with a co-factor (PDB ID: $3 \mathrm{kb6}$ ) corresponds to aq_727 from Aquifex aeolicus and is annotated as D-lactate dehydrogenase in both PDB and UniProt. X9 is a sister group to LDHD (Fig. 1), although representatives of the two subfamilies display relatively low sequence identity $(<40 \%)$. $3 \mathrm{~kb} 6$ was determined in complex with $\mathrm{NAD}(\mathrm{H})$ and lactic acid (the authors suggested that it could also be pyruvate, but the electron density clearly suggests that the $\mathrm{C} 2$ atom of this molecule is $\mathrm{sp}^{3}$ hybridized) [41], which interacts with the residues in a similar fashion as those seen in LDHD structures (e.g., PDB ID: 4cuk, Additional file 8: Figure S4). Therefore, despite a lack of biochemical evidence, X9 may be regarded as a new subgroup of D-lactate dehydrogenases.

\section{HADHs knowledgebase}

To simplify navigation over the $2 \mathrm{HADH}$ classification, we have created a software tool that converts spreadsheets containing results of our analyses into a web-based knowledgebase. The $2 \mathrm{HADHs}$ knowledgebase consists of three elements: an explorable phylogenetic tree of the family, an interactive table with annotations of the selected enzymes, and a BLAST search tool. Main clades on the phylogenetic tree are hyperlinked to the table with annotations of the family representatives. Protein annotations include a list of kinetically characterized substrates, highest efficiency substrate, PDB identifiers, structure ligands, and publication references for structural and kinetics studies. The protein table content can be sorted by any column and filtered by source organism kingdom, availability of kinetics, or structural studies. All proteins that have the corresponding publication or an experimentally-determined structure are hyperlinked to PubMed and the Protein Data Bank, respectively. In addition, we have generated Molstack [95] visualizations of active sites for all proteins having a cofactor and a ligand bound in this region. Molstack interactive visualizations give an instant insight into the quality of macromolecule model and a corresponding electron density map. The knowledgebase allows to classify an uncharacterized sequence and find its closest studied homologs by using a built-in BLAST tool. Its content is generated automatically from annotation spreadsheets, what makes it is easy to maintain the information up-to-date. The 2HADHs knowledgebase is publicly available at http://2hadh.bioreproducibility.org/.

\section{Discussion}

Motivated by recent advances in genetic engineering and new societal needs, the use of enzymes as catalysts to synthesize compounds and materials is rapidly expanding. It is apparent that enzyme promiscuity offers great opportunities in the design and development of new catalytic functions in the scaffold of stable enzymes [96]. Exploiting enzyme substrate promiscuity might lead to improvements in existing catalysts and provide novel synthesis pathways that are currently not available. The D-2-hydroxyacid dehydrogenases (2HADHs) may be considered as another protein family in which substrate promiscuity and moderate-to-high efficiency are a rule rather than an exception. This feature has already been used in systems for highly stereoselective production of 
selected chiral $\alpha$-hydroxy carboxylic acids $[11,12]$. The comprehensive understanding of sequence-structurefunction information provides a foundation for future biotechnological developments.

A refined evolutionary analysis and classification of the $2 \mathrm{HADH}$ family to large extent uphold the previous observation that most enzymes remain to cluster according to their preferential substrates. Moreover, the topology of the refined tree suggests that, besides previously determined six subfamilies (SERA, FDH, CTBP, PDXB, LDHD, and GHRB), three others (GHRA, GHRC, and $\mathrm{DDH}$ ) with at least one biochemically characterized member could be established. Moreover, we define 13 other small subfamilies of dehydrogenases that have not been characterized biochemically. We suggest that studying representatives of these subfamilies can greatly help annotation of metabolic pathways of multiple organisms, as well as may lead to discovery of enzymes with novel biotechnological applications.

To facilitate application of the collected information, we provide a publicly available $2 \mathrm{HADH}$ enzymes knowledge portal, which allows to classify uncharacterized members and gives insights into the evolutionary history of substrate specificity of these heterogeneous enzymes. Although several databases have been developed to store kinetic parameters of enzymes obtained in steady states (e.g., BRENDA [97], MetaCyc [98], or UniProt [99]), for a majority of the protein families, the data are usually sparse and encompass only subsets of possible substrates. Therefore, to limit the bias and grasp the level to which the functional annotations can be extra- or interpolated, the data should be interpreted in the evolutionary context of the whole protein family. The large number of paralogs, recent duplications and horizontal transfers make function prediction within the $2 \mathrm{HADH}$ family particularly troublesome. To facilitate usage of the collected information, the $2 \mathrm{HADH}$ portal is equipped with an interface to search for close homologs within the representative enzymes and an interactive annotation data table. The portal is designed to be easily maintained and adaptable to the presentation of similar analysis of other protein families. The $2 \mathrm{HADH}$ knowledgebase is available at: http://2hadh.bioreproducibility.org/.

\section{Conclusions}

We present a revised classification of the family that comprises 22 subfamilies, including 13 new subfamilies not studied biochemically. For the first time, all available enzymatic and structural features of the subfamilies were collected and analyzed in a systematic way, expanding our understanding of the features contributing to their core function of D-2-hydroxyacid dehydrogenation, as well as to their functional diversity exhibited by substrate specificities. Our family-wide sequence and structural comparison proved general importance of several active site residues that were not previously discussed in the literature (e.g., Val72, Gly73, Asn227, Gly229, and Gly231, with numbering referring to Q92LZ4_RHIME), extending our understanding of its catalytic machinery. Systematic analyses of active site environments provided key insights into the residues important (or unimportant) for substrate selectivity. In addition, these analyses have left intriguing uncertainties regarding the role of dimerization and dynamics of the secondary structure elements or entire domains, as well as the molecular mechanism for different substrate ambiguity. To facilitate usage of the collected biochemical, structural and evolutionary information, we provide a dedicated web portal allowing to classify new sequences and to generate functional hypotheses for further studies on these largely uncharacterized enzymes.

\section{Methods \\ Phylogenetic analysis}

To reliably classify the $2 \mathrm{HADH}$ family into evolutionary subfamilies, we constructed a phylogenetic tree using available sequence and structure information. Phylogenetic tree building relies primarily on a multiple sequence alignment (MSA) of sequences of interest. Standard automatic algorithms for building MSAs do not work well for sequences with low sequence identity, i.e., below $20-25 \%$ [100], but could be considerably improved by incorporating structural information [101]. For example, sequence identity between $A$. thaliana $\mathrm{FDH}$ and $\mathrm{H}$. sapiens CTBP1 is $18 \%$, as obtained from a global Needleman-Wunsch alignment. Given that the existing $2 \mathrm{HADH}$ classification relies on an MSA generated automatically with ClustalW $[5,6]$, we decided to improve it by using a high-quality, structure-based MSA.

First, literature searches were carried out to identify and select biochemically confirmed D-2-hydroxyacid dehydrogenases. The biochemically studied proteins were used as a "confidently annotated" reference set for the $2 \mathrm{HADH}$ family. Their amino acid sequences were downloaded from UniProt, trimmed to the cofactor-binding and substrate-binding domains, and used as queries for BLAST [102] against the PDB (expectation value $<10^{-5}$, September 2016) to search for closely homologous enzymes with solved structures. 30 selected PDB representatives were used to create a high-quality, structure-based sequence alignment with PROMALS3D [101] using the default parameters. The "seed" structures were chosen to diversely represent the $2 \mathrm{HADH}$ sequence space (median identity between sequences was $23 \%$ ).

To extend the sequence set, the reference $2 \mathrm{HADH}$ enzymes with biochemical or structural information was then used as queries in BLAST searches against 111 
representative proteomes downloaded from the KEGG GENOME (Additional file 9: Data file S2). Hits with $E$-value $<10^{-3}$ to at least one reference sequence and with coverage at least $90 \%$ of the query sequence were extracted and aligned with MAFFT 7.123 [100] ("mafft-linsi --add") to the structure-based alignment of the representative structures. The alignment of 462 sequences was manually checked in SeaView 4.5.4 [103], and trimmed with trimAl to remove columns with gaps in at least $80 \%$ sequences ("trimal -gt 0.2") [104].

Based on the resulting MSA of 462 sequences, we built phylogenetic trees using several approaches. Phylogenetic inference was carried out using neighbor-joining (NJ) and maximum-likelihood (ML) methods. The NJ tree was calculated with BioNJ [105] (Poisson distance, 100 bootstrap replicas, and JTT model). The ML trees were computed with FastTree 2.1.7 [106] (WAG+CAT evolutionary model, discrete gamma model with 20 rate categories and Shimodaira-Hasegawa test for estimation of local support values) and RAxML 8.2.7 [107] (100 bootstrap replicas, WAG evolutionary model, and estimated gamma distribution parameter: "-T 100 -f a $-\mathrm{m}$ PROTGAMMAWAG -p 12345 -x 12345 -\# 100"). The obtained trees were visualized with Archaeopteryx [108].

\section{Structure analysis}

Crystal structures deposited in the PDB were collected with BLAST via the RCSB PDB RESTful interface [109], using sequences of the functionally annotated 2HADHs as queries (with E-value threshold of $10^{-5}$ ). The structures were then analyzed with BioPython [110] and PyMol [111]. The complete list of the analyzed structures can be found in Additional file 6: Table S2.

In the 40 structures solved with both a cofactor and a ligand analog bound in the active site, we mapped residues within $5 \AA$ from the bound substrate (Additional file 8: Figure S4).

\section{Web server}

The web server was created in JavaScript and Node.js run-time environment. The BLAST database of the 462 sequences was generated with "makeblastdb". Sequence searching is carried out using "blastp" command with default parameters [102]. The web server is accessible at http://2hadh.bioreproducibility.org/.

\section{Additional files}

Additional file 1: Supplementary Results. Horizontal gene transfer from bacteria to plants. (PDF $28 \mathrm{~kb}$ )

Additional file 2: Figure S1. Maximum-likelihood evolutionary tree of the $2 \mathrm{HADH}$ family. The branch labels correspond to UniProt accessions of proteins with studied substrate specificities (orange dots), known crystal structures (green dots), or both (red dots). The scale bar represents the number of estimated changes per position. A crystal structure of GHRC from Desulfovibrio vulgaris (PDB ID: $5 \mathrm{t} \times 7$ ) was solved after the analysis was performed, and is not shown in the figure. (PDF $56 \mathrm{~kb}$ )

Additional file 3: Data file S1. Phylogenetic trees in Newick format. In the order of appearance in the file: 1) maximum-likelihood tree computed with FastTree, 2) maximum-likelihood tree computed with RAxML, 3) neighbor-joining tree computed with BioNJ. Node names refer to either KEGG or UniProt accessions. (TXT $59 \mathrm{~kb}$ )

Additional file 4: Table S1. Kinetic parameters for $2 \mathrm{HADH}$ and prominent substrates described in the literature. (XLSX 503 kb)

Additional file 5: Figure S2. Kinetic parameters $\left(a, k_{\text {cat }} / K_{M} ; b, K_{M}\right)$ for $2 \mathrm{HADH}$ from the nine biochemically studies subfamilies. Results are illustrated as box-and-whisker plots where the thick line represents the median within the subfamilies and the box area encompasses 50\% of all observations. Red dots correspond to the most efficient substrates (in terms of $k_{\text {cat }} / K_{M}$ or relative catalytic activity) for the enzymes, grey dots to secondary substrates. (PDF $71 \mathrm{~kb}$ )

Additional file 6: Table S2. Table of $2 \mathrm{HADH}$ s of known crystal structure. (XLSX $495 \mathrm{~kb}$ )

Additional file 7: Figure S3. Sequence logos of all defined $2 \mathrm{HADH}$ subfamilies aligned with the reference sequence of Rhizobium meliloti GHRB (Q92LZ4). The structure-based alignment was obtained for selected structures with PROMALS3D and used as a seed alignment for other $2 \mathrm{HADH}$ sequences from 111 representative organisms. The sequence logos were generated with WebLogo, showing columns for which in at least one subfamily at least $90 \%$ members possess an amino acid (i.e., with at most $10 \%$ gapped positions). C-terminal fragments were cut out, except for the fragment of the PDXB dimerization domain ("PDXB-dim"). The top row denotes secondary structure elements common for the substrate-binding (i.e., a1-a5 and $\beta 1-\beta 5$ ) and catalytic (i.e., aA-aH and $\beta A$ $\beta G)$ domains. The bottom row indicates regions potentially involved in substrate binding, with catalytic triad residues denoted by red triangles. (PDF $1582 \mathrm{~kb}$ )

Additional file 8: Figure S4. Active sites of selected $2 \mathrm{HADH}$ enzymes. Cofactors, substrates (or their analogs), and residues that potentially contribute to substrate specificity are shown as sticks. Carbon atoms of substrates or their analogs are shown in black. Colors of the residue labels correspond to structural regions of the proteins (see also Fig. 3). The residues of the catalytic triad are indicated with red labels. (PDF $9523 \mathrm{~kb}$ )

Additional file 9: Data file S2. List of KEGG organisms with completely sequenced genomes used for protein sequence searches. (TXT 8 kb)

\section{Abbreviations}

2HADH: D-2-hydroxyacid dehydrogenase; CTBP: C-terminal binding proteins; DDH: broad-substrate-specificity dehydrogenases; FDH: formate dehydrogenases; GHRA: glyoxylate/hydroxypyruvate reductases A; GHRB: glyoxylate/hydroxypyruvate reductases B; GHRC: glyoxylate/ hydroxypyruvate reductases C; GRHPR: human glyoxylate reductase; $K_{1 / 2}$ : the concentration of substrate that produces a half-maximal enzyme velocity under the sigmoidal model of kinetics; $k_{c a t}$ : turnover number; $K_{M}$ : Michaelis constant, i.e., the concentration of substrate that produces a half-maximal enzyme velocity under the Michaelis-Menten model; LDHD: D-lactate dehydrogenases; ML: maximum-likelihood; MSA: multiple sequence alignment; $\mathrm{NAD}^{+}$: nicotinamide adenine dinucleotide; $\mathrm{NADP}^{+}$: Nicotinamide adenine dinucleotide phosphate; NJ: neighbor-joining; PDB: Protein Data Bank; PDXB: 4-phosphoerythronate dehydrogenases; SERA: 3phosphoglycerate dehydrogenases; $\mathrm{X} 1-\mathrm{X} 13$ : new $2 \mathrm{HADH}$ subfamilies

\section{Acknowledgements}

We thank Tomasz Osinski and Marek Grabowski for their help in setting up and maintaining the $2 \mathrm{HADH}$ knowledgebase server. We thank Misty Kuhn for helpful discussions. We would also like to thank Matthew Zimmerman, Barat S. Venkataramany, and David Cooper for critical readings of the manuscript and valuable comments.

\section{Funding}

This project was funded by the National Institute of Allergy and Infectious Diseases, National Institutes of Health (NIH), U.S. Department of Health and 
Human Services, under Contracts No. HHSN272201200026C and HHSN272201700060C (CSGID); NIH Big Data to Knowledge (BD2K) grant HG008424; NIGMS grants No. U54-GM094662 and GM118619; and by the Foundation for Polish Science (TEAM) and Polish National Science Centre (2014/15/B/NZ1/03357) grants to K.G. The funders had no role in study design, data collection and analysis, and preparation of the manuscript.

\section{Availability of data and materials}

The datasets supporting the conclusions of this article are available within its additional files, as well as in the 2HADH knowledgebase website, http:// 2hadh.bioreproducibility.org/.

\section{Authors' contributions}

Conceived and designed the experiments: DM IGS WM. Performed the experiments: DM JJ. Analyzed the data: DM JJ IGS JK KG WM. Created the web server: DM MD. Wrote the paper: DM IGS. Edited and revised the paper: DM IGS MD KG WM. All authors read and approved the final manuscript.

\section{Ethics approval and consent to participate}

Not applicable.

\section{Consent for publication}

Not applicable.

\section{Competing interests}

The authors declare that they have no competing interests.

\section{Publisher's Note}

Springer Nature remains neutral with regard to jurisdictional claims in published maps and institutional affiliations.

\section{Author details}

'Department of Molecular Physiology and Biological Physics, University of Virginia, 1340 Jefferson Park Avenue, Charlottesville, VA 22908, USA. ${ }^{2}$ Laboratory of Bioinformatics and Systems Biology, Centre of New Technologies, University of Warsaw, Zwirki i Wigury 93, 02-089 Warsaw, Poland. ${ }^{3}$ Center for Structural Genomics of Infectious Diseases (CSGID), Charlottesville, VA 22908, USA. " Laboratory for Structural and Biochemical Research, Biological and Chemical Research Centre, Department of Chemistry, University of Warsaw, Zwirki i Wigury 101, 02-089 Warsaw, Poland. ${ }^{5}$ Department of Chemistry, University of Warsaw, Ludwika Pasteura 1, 02-093 Warsaw, Poland.

\section{Received: 9 July 2018 Accepted: 27 November 2018}

\section{Published online: 22 December 2018}

\section{References}

1. Stoll VS, Manohar AV, Gillon W, MacFarlane EL, Hynes RC, Pai EF. A thioredoxin fusion protein of $\mathrm{VanH}$, a D-lactate dehydrogenase from enterococcus faecium: cloning, expression, purification, kinetic analysis, and crystallization. Protein Sci. 1998;7(5):1147-55.

2. Kleczkowski LA, Randall DD. Purification and characterization of a novel $\mathrm{NADPH}(\mathrm{NADH})$-dependent hydroxypyruvate reductase from spinach leaves. Comparison of immunological properties of leaf hydroxypyruvate reductases. Biochem J. 1988;250(1):145-52.

3. Tarmy E, Kaplan N. Kinetics of Escherichia coli B D-lactate dehydroge and evidence for pyruvate-controlled change in conformation. J Biol Chem. 1968;243(10):2587-96.

4. Cramer SD, Ferree PM, Lin K, Milliner DS, Holmes RP. The gene encoding hydroxypyruvate reductase (GRHPR) is mutated in patients with primary hyperoxaluria type II. Hum Mol Genet. 1999;8(11):2063-9.

5. Fauvart M, Braeken K, Daniels R, Vos K, Ndayizeye M, Noben JP, Robben J, Vanderleyden J, Michiels J. Identification of a novel glyoxylate reductase supports phylogeny-based enzymatic substrate specificity prediction. Biochim Biophys Acta. 2007;1774(9):1092-8.

6. Fujii T, Shimizu M, Doi Y, Fujita T, Ito T, Miura D, Wariishi H, Takaya N. Novel fungal phenylpyruvate reductase belongs to D-isomer-specific 2hydroxyacid dehydrogenase family. Biochim Biophys Acta. 2011;1814(12): 1669-76.

7. Wada Y, Iwai S, Tamura Y, Ando T, Shinoda T, Arai K, Taguchi H. A new family of D-2-hydroxyacid dehydrogenases that comprises D-mandelate dehydrogenases and 2-ketopantoate reductases. Biosci Biotechnol Biochem. 2008;72(4):1087-94.

8. Zheng Z, Ma C, Gao C, Li F, Qin J, Zhang H, Wang K, Xu P. Efficient conversion of phenylpyruvic acid to phenyllactic acid by using whole cells of Bacillus coagulans SDM. PLoS One. 2011;6(4):e19030.

9. Gao C, Zhang W, Ma C, Liu P, Xu P. Kinetic resolution of 2hydroxybutanoate racemic mixtures by NAD-independent L-lactate dehydrogenase. Bioresour Technol. 2011;102(7):4595-9.

10. Chen B, Yin HF, Wang ZS, Liu JY, Xu JH. A new chemo-enzymatic route to chiral 2-hydroxy-4-phenylbutyrates by combining lactonase-mediated resolution with hydrogenation over Pd/C. Chem Commun (Camb). 2010; 46(16):2754-6.

11. Zheng Z, Sheng B, Gao C, Zhang H, Qin T, Ma C, Xu P. Highly stereoselective biosynthesis of (R)-alpha-hydroxy carboxylic acids through rationally re-designed mutation of D-lactate dehydrogenase. Sci Rep. 2013;3: 3401

12. Sheng B, Zheng Z, Lv M, Zhang H, Qin T, Gao C, Ma C, Xu P. Efficient production of (R)-2-hydroxy-4-phenylbutyric acid by using a coupled reconstructed D-lactate dehydrogenase and formate dehydrogenase system. PLoS One. 2014;9(8):e104204.

13. Shaked ZE, Whitesides GM. Enzyme-catalyzed organic synthesis: NADH regeneration by using formate dehydrogenase. J Am Chem Soc. 1980; 102(23):7104-5.

14. Berrios-Rivera SJ, Bennett GN, San KY. Metabolic engineering of Escherichia coli: increase of NADH availability by overexpressing an NAD (+)-dependent formate dehydrogenase. Metab Eng. 2002;4(3):217-29.

15. Choe H, Joo JC, Cho DH, Kim MH, Lee SH, Jung KD, Kim YH. Efficient CO2reducing activity of NAD-dependent formate dehydrogenase from Thiobacillus sp. KNK65MA for formate production from CO2 gas. PLoS One. 2014;9(7):e103111.

16. Tobey KL, Grant GA. The nucleotide sequence of the serA gene of Escherichia coli and the amino acid sequence of the encoded protein, D-3phosphoglycerate dehydrogenase. J Biol Chem. 1986;261(26):12179-83.

17. Schoenlein PV, Roa BB, Winkler ME. Divergent transcription of pdxB and homology between the pdxB and serA gene products in Escherichia coli K12. J Bacteriol. 1989;171(11):6084-92.

18. Davies TJ, Barraclough TG, Chase MW, Soltis PS, Soltis DE, Savolainen V. Darwin's abominable mystery: insights from a supertree of the angiosperms. Proc Natl Acad Sci U S A. 2004;101(7):1904-9.

19. Chistoserdova LV, Lidstrom ME. Purification and characterization of hydroxypyruvate reductase from the facultative methylotroph Methylobacterium extorquens AM1. J Bacteriol. 1991;173(1):7228-32.

20. Williamson DH, Lund P, Krebs HA. The redox state of free nicotinamideadenine dinucleotide in the cytoplasm and mitochondria of rat liver. Biochem J. 1967;103(2):514-27.

21. Tishkov VI, Popov VO. Catalytic mechanism and application of formate dehydrogenase. Biochemistry (Mosc). 2004;69(11):1252-67.

22. Bar-Even A, Noor E, Savir Y, Liebermeister W, Davidi D, Tawfik DS, Milo R. The moderately efficient enzyme: evolutionary and physicochemical trends shaping enzyme parameters. Biochemistry. 2011;50(21):4402-10.

23. Costas AM, White AK, Metcalf WW. Purification and characterization of a novel phosphorus-oxidizing enzyme from Pseudomonas stutzeri WM88. J Biol Chem. 2001;276(20):17429-36.

24. Baker DP, Kleanthous C, Keen JN, Weinhold E, Fewson CA. Mechanistic and active-site studies on D(--)-mandelate dehydrogenase from Rhodotorula graminis. Biochem J. 1992;281:211-8.

25. Hatrongjit R, Packdibamrung K. A novel NADP+-dependent formate dehydrogenase from Burkholderia stabilis 15516: screening, purification and characterization. Enzym Microb Technol. 2010;46(7):557-61.

26. Khersonsky O, Tawfik DS. Enzyme promiscuity: a mechanistic and evolutionary perspective. Annu Rev Biochem. 2010;79:471-505.

27. Rudolph J, Kim J, Copley SD. Multiple turnovers of the nicotino-enzyme PdxB require a-keto acids as cosubstrates. Biochemistry. 2010;49(43): 9249-55.

28. Dengler U, Niefind K, Kieß M, Schomburg D. Crystal structure of a ternary complex of D-2-hydroxyisocaproate dehydrogenase from lactobacillus casei, NAD+ and 2-oxoisocaproate at $1.9 \AA$ resolution. J Mol Biol. 1997;267(3):640-60.

29. Razeto A, Kochhar S, Hottinger H, Dauter M, Wilson KS, Lamzin VS. Domain closure, substrate specificity and catalysis of D-lactate dehydrogenase from lactobacillus bulgaricus. J Mol Biol. 2002;318(1):109-19. 
30. Shabalin IG, Filippova EV, Polyakov KM, Sadykhov EG, Safonova TN, Tikhonova TV, Tishkov VI, Popov VO. Structures of the apo and holo forms of formate dehydrogenase from the bacterium Moraxella sp. C-1: towards understanding the mechanism of the closure of the interdomain cleft. Acta Crystallogr D Biol Crystallogr. 2009;65:1315-25.

31. Wierenga RK, Terpstra P, Hol WG. Prediction of the occurrence of the ADPbinding beta alpha beta-fold in proteins, using an amino acid sequence fingerprint. J Mol Biol. 1986;187(1):101-7.

32. Bellamacina CR. The nicotinamide dinucleotide binding motif: a comparison of nucleotide binding proteins. FASEB J. 1996;10(11):1257-69.

33. Alekseeva AA, Fedorchuk W, Zarubina SA, Sadykhov EG, Matorin AD, Savin SS, Tishkov VI. The role of ala198 in the stability and coenzyme specificity of bacterial formate dehydrogenases. Acta Nat. 2015;7(1):60-9.

34. Ha JY, Lee JH, Kim KH, Kim DJ, Lee HH, Kim HK, Yoon HJ, Suh SW. Crystal structure of D-erythronate-4-phosphate dehydrogenase complexed with NAD. J Mol Biol. 2007;366(4):1294-304.

35. Lamzin VS, Dauter Z, Popov VO, Harutyunyan EH, Wilson KS. High resolution structures of holo and apo formate dehydrogenase. J Mol Biol. 1994;236(3): 759-85.

36. Bernard N, Johnsen K, Holbrook JJ, Delcour J. D175 discriminates between $\mathrm{NADH}$ and NADPH in the coenzyme binding site of lactobacillus delbrueckii subsp. bulgaricus D-lactate dehydrogenase. Biochem Biophys Res Commun. 1995;208(3):895-900.

37. Lamzin VS, Dauter Z, Wilson KS. Dehydrogenation through the lookingglass. Nat Struct Biol. 1994;1(5):281-2.

38. Booth MPS, Conners R, Rumsby G, Brady RL. Structural basis of substrate specificity in human glyoxylate reductase/hydroxypyruvate reductase. J Mol Biol. 2006;360(1):178-89.

39. Holton SJ, Anandhakrishnan M, Geerlof A, Wilmanns M. Structural characterization of a D-isomer specific 2-hydroxyacid dehydrogenase from lactobacillus delbrueckii ssp. bulgaricus J Struct Biol. 2013;181(2):179-84.

40. Hilbert BJ, Grossman SR, Schiffer CA, Royer WE. Crystal structures of human CtBP in complex with substrate MTOB reveal active site features useful for inhibitor design. FEBS Lett. 2014;588(9):1743-8.

41. Antonyuk SV, Strange RW, Ellis MJ, Bessho Y, Kuramitsu S, Inoue Y, Yokoyama S, Hasnain SS. Structure of D-lactate dehydrogenase from Aquifex aeolicus complexed with NAD+ and lactic acid (or pyruvate). Acta Crystallogr Sect F Struct Biol Cryst Commun. 2009;65:1209-13.

42. Shabalin IG, Porebski PJ, Minor W. Refining the macromolecular model achieving the best agreement with the data from X-ray diffraction experiment. Crystallogr Rev. 2018;24(4):236-62.

43. Taguchi H, Ohta T. Essential role of arginine 235 in the substratebinding of lactobacillus plantarum D-lactate dehydrogenase. J Biochem. 1994;115(5):930-6.

44. Taguchi H, Ohta T, Matsuzawa H. Involvement of Glu-264 and Arg-235 in the essential interaction between the catalytic imidazole and substrate for the D-lactate dehydrogenase catalysis. J Biochem. 1997;122(4):802-9.

45. Popov VO, Tishkov VI. NAD+ -dependent formate dehydrogenase. From a model enzyme to a versatile biocatalyst. Research Signpost. 2003;661:345-69.

46. Tishkov VI, Matorin AD, Rojkova AM, Fedorchuk W, Savitsky PA, Dementieva LA, Lamzin VS, Mezentzev AV, Popov VO. Site-directed mutagenesis of the formate dehydrogenase active Centre: role of the His332-Gln313 pair in enzyme catalysis. FEBS Lett. 1996;390(1):104-8.

47. Singh RK, Raj I, Pujari R, Gourinath S. Crystal structures and kinetics of type III 3-phosphoglycerate dehydrogenase reveal catalysis by lysine. FEBS J. 2014;281(24):5498-512.

48. Shinoda T, Arai K, Shigematsu-lida M, Ishikura Y, Tanaka S, Yamada T, Kimber MS, Pai EF, Fushinobu S, Taguchi H. Distinct conformation-mediated functions of an active site loop in the catalytic reactions of NAD-dependent D-lactate dehydrogenase and formate dehydrogenase. J Biol Chem. 2005; 280:17068-75.

49. Winn PJ, Ludemann SK, Gauges R, Lounnas V, Wade RC. Comparison of the dynamics of substrate access channels in three cytochrome P450s reveals different opening mechanisms and a novel functional role for a buried arginine. Proc Natl Acad Sci U S A. 2002;99(8):5361-6.

50. Tokuriki N, Tawfik DS. Protein dynamism and evolvability. Science. 2009; 324(5924):203-7.

51. Nibu $Y$, Zhang $H$, Levine $M$. Interaction of short-range repressors with Drosophila CtBP in the embryo. Science. 1998;280(5360):101-4.

52. Chinnadurai G. CtBP, an unconventional transcriptional corepressor in development and oncogenesis. Mol Cell. 2002;9(2):213-24.
53. Sewalt RG, Gunster MJ, van der Vlag J, Satijn DP, Otte AP. C-terminal binding protein is a transcriptional repressor that interacts with a specific class of vertebrate Polycomb proteins. Mol Cell Biol. 1999;19(1):777-87.

54. Turner J, Crossley M. The CtBP family: enigmatic and enzymatic transcriptional co-repressors. BioEssays. 2001;23(8):683-90.

55. Marmorstein R. Dehydrogenases, NAD, and transcription--what's the connection? Structure. 2002;10(11):1465-6.

56. Kumar V, Carlson JE, Ohgi KA, Edwards TA, Rose DW, Escalante CR, Rosenfeld MG, Aggarwal AK. Transcription corepressor CtBP is an NAD+ -regulated dehydrogenase. Mol Cell. 2002;10(4):857-69.

57. Zhang Q, Piston DW, Goodman RH. Regulation of corepressor function by nuclear NADH. Science. 2002;295(5561):1895-7.

58. Achouri Y, Noël G, Van Schaftingen E. 2-Keto-4-methylthiobutyrate, an intermediate in the methionine salvage pathway, is a good substrate for CtBP1. Biochem Biophys Res Commun. 2007;352(4):903-6.

59. Tsukaya $H$. A new member of the CtBP/BARS family from plants: Angustifolia. In: Madame Curie Bioscience Database. Landes Bioscience: Austin (TX); 2013.

60. Thio SS, Bonventre JV, Hsu SI. The CtBP2 co-repressor is regulated by NADHdependent dimerization and possesses a novel $\mathrm{N}$-terminal repression domain. Nucleic Acids Res. 2004;32(5):1836-47.

61. Madison DL, Wirz JA, Siess D, Lundblad JR. Nicotinamide adenine dinucleotide-induced multimerization of the co-repressor CtBP1 relies on a switching tryptophan. J Biol Chem. 2013;288(39):27836-48.

62. Domenech J, Ferrer J. A new D-2-hydroxyacid dehydrogenase with dual coenzyme-specificity from Haloferax mediterranei, sequence analysis and heterologous overexpression. Biochim Biophys Acta. 2006;1760(11):1667-74.

63. Popov V, Lamzin V. NAD+ -dependent formate dehydrogenase. Biochem J. 1994;301:625-43.

64. Fogal S, Beneventi E, Cendron L, Bergantino E. Structural basis for double cofactor specificity in a new formate dehydrogenase from the acidobacterium Granulicella mallensis MP5ACTX8. Appl Microbiol Biotechnol. 2015;99(22):9541-54.

65. Guo Q, Gakhar L, Wickersham K, Francis K, Vardi-Kilshtain A, Major DT, Cheatum CM, Kohen A. Structural and kinetic studies of formate dehydrogenase from Candida boidinii. Biochemistry. 2016;55(19):2760-71.

66. Zhao G, Pease AJ, Bharani N, Winkler ME. Biochemical characterization of gapB-encoded erythrose 4-phosphate dehydrogenase of Escherichia coli K12 and its possible role in pyridoxal 5'-phosphate biosynthesis. J Bacteriol. 1995;177(10):2804-12.

67. Nilov DK, Shabalin IG, Popov VO, Svedas VK. Investigation of formate transport through the substrate channel of formate dehydrogenase by steered molecular dynamics simulations. Biochemistry (Mosc). 2011;76(2): $172-4$.

68. Nilov DK, Shabalin IG, Popov VO, Svedas VK. Molecular modeling of formate dehydrogenase: the formation of the Michaelis complex. J Biomol Struct Dyn. 2012;30(2):170-9.

69. Nuñez MF, Pellicer MT, Badia J, Aguilar J, Baldoma L. Biochemical characterization of the 2-ketoacid reductases encoded by ycdW and yiaE genes in Escherichia coli. Biochem J. 2001;354:707-15.

70. Kutner J, Shabalin IG, Matelska D, Handing K, Gasiorowska O, Sroka P, Gorna MW, Ginalski K, Wozniak K, Minor W. Structural, biochemical, and evolutionary characterization of glyoxylate/hydroxypyruvate reductases shows their division into two distinct subfamilies. Biochemistry. 2018;57(6): 963-77.

71. Carugo O, Argos P. NADP-dependent enzymes. I: conserved stereochemistry of cofactor binding. Proteins. 1997;28(1):10-20.

72. Lassalle L, Engilberge S, Madern D, Vauclare P, Franzetti B, Girard E. New insights into the mechanism of substrates trafficking in Glyoxylate/ Hydroxypyruvate reductases. Sci Rep. 2016;6:20629.

73. Kim KH, Janiak V, Petersen M. Purification, cloning and functional expression of hydroxyphenylpyruvate reductase involved in rosmarinic acid biosynthesis in cell cultures of Coleus blumei. Plant Mol Biol. 2004; 54(3):311-23.

74. Timm S, Nunes-Nesi A, Pärnik T, Morgenthal K, Wienkoop S, Keerberg O, Weckwerth W, Kleczkowski LA, Fernie AR, Bauwe H. A cytosolic pathway for the conversion of hydroxypyruvate to glycerate during photorespiration in Arabidopsis. Plant Cell. 2008;20(10):2848-59.

75. Miyazaki SS, Toki S, Izumi Y, Yamada H. Purification and characterization of a serine hydroxymethyltransferase from an obligate methylotroph, Hyphomicrobium methylovorum GM2. Eur J Biochem. 1987;162(3):533-40. 
76. Yum DY, Lee BY, Hahm DH. The yiaE gene, located at 80.1 minutes on the Escherichia coli chromosome, encodes a 2-ketoaldonate reductase. J Bacteriol. 1998;180(22):5984-8.

77. Li Y, Tian CF, Chen WF, Wang L, Sui XH, Chen WX. High-resolution transcriptomic analyses of Sinorhizobium sp. NGR234 bacteroids in determinate nodules of Vigna unguiculata and indeterminate nodules of Leucaena leucocephala. PLoS One. 2013;8(8):e70531.

78. Johannes TW, Woodyer RD, Zhao H. Directed evolution of a thermostable phosphite dehydrogenase for NAD (P) H regeneration. Appl Environ Microbiol. 2005;71(10):5728-34

79. Zou Y, Zhang H, Brunzelle JS, Johannes TW, Woodyer R, Hung JE, Nair N, Van Der Donk WA, Zhao H, Nair SK. Crystal structures of phosphite dehydrogenase provide insights into nicotinamide cofactor regeneration. Biochemistry. 2012;51(21):4263-70.

80. Hung JE, Fogle EJ, Christman HD, Johannes TW, Zhao H, Metcalf WW, van der Donk WA. Investigation of the role of Arg301 identified in the X-ray structure of phosphite dehydrogenase. Biochemistry. 2012;51(21):4254-62

81. Lapierre L, Germond JE, Ott A, Delley M, Mollet B. D-lactate dehydrogenase gene (ldhD) inactivation and resulting metabolic effects in the lactobacillus johnsonii strains La1 and N312. Appl Environ Microbiol. 1999;65(9):4002-7.

82. Bugg TD, Wright GD, Dutka-Malen S, Arthur M, Courvalin P, Walsh CT. Molecular basis for vancomycin resistance in enterococcus faecium BM4147: biosynthesis of a depsipeptide peptidoglycan precursor by vancomycin resistance proteins VanH and VanA. Biochemistry. 1991;30(43):10408-15.

83. Burgess SJ, Taha H, Yeoman JA, lamshanova O, Chan KX, Boehm M, Behrends V, Bundy JG, Bialek W, Murray JW, et al. Identification of the elusive pyruvate reductase of Chlamydomonas reinhardtii chloroplasts. Plant Cell Physiol. 2016;57(1):82-94

84. Kallwass HKW. Potential of R-2-Hydroxyisocaproate dehydrogenase from lactobacillus casei for stereospecific reductions. Enzym Microb Technol. 1992;14(1):28-35.

85. Busto F, Soler J, Arriaga DD, Cadenas E. In situ behaviour of D (-)-lactate dehydrogenase from Escherichia coli. Arch Microbiol. 1984;139(2-3):255-9.

86. Grant GA. Contrasting catalytic and allosteric mechanisms for phosphoglycerate dehydrogenases. Arch Biochem Biophys. 2012;519(2): 175-85.

87. Dey S, Hu Z, Xiao LX, Sacchettini JC, Grant GA. D-3-phosphoglycerate dehydrogenase from Mycobacterium tuberculosis is a link between the Escherichia coli and mammalian enzymes. J Biol Chem. 2005;280(15): 14884-91.

88. Grant GA. The ACT domain: a small molecule binding domain and its role as a common regulatory element. J Biol Chem. 2006;281(45):33825-9.

89. Xu XL, Grant GA. Regulation of Mycobacterium tuberculosis D-3phosphoglycerate dehydrogenase by phosphate-modulated quaternary structure dynamics and a potential role for polyphosphate in enzyme regulation. Biochemistry. 2014;53(26):4239-49.

90. Schuller D, Grant G, Banaszak L. The allosteric ligand site in the Vmax-type. Nat Struct Biol. 1995;2(1):69-76

91. Sugimoto E, Pizer LI. The mechanism of end product inhibition of serine biosynthesis. I. Purification and kinetics of phosphoglycerate dehydrogenase. J Biol Chem. 1968;243(9):2081-9.

92. Zhao G, Winkler ME. A novel alpha-ketoglutarate reductase activity of the serA-encoded 3-phosphoglycerate dehydrogenase of Escherichia coli K-12 and its possible implications for human 2-hydroxyglutaric aciduria. J Bacteriol. 1996;178(1):232-9.

93. Ali V, Hashimoto T, Shigeta Y, Nozaki T. Molecular and biochemical characterization of D-phosphoglycerate dehydrogenase from Entamoeba histolytica: a unique enteric protozoan parasite that possesses both phosphorylated and nonphosphorylated serine metabolic pathways. Eur J Biochem. 2004:271(13):2670-81.

94. Denger K, Cook AM. Racemase activity effected by two dehydrogenases in sulfolactate degradation by Chromohalobacter salexigens: purification of (S)sulfolactate dehydrogenase. Microbiology. 2010;156:967-74.

95. Porebski PJ, Sroka P, Zheng H, Cooper DR, Minor W. Molstack-interactive visualization tool for presentation, interpretation, and validation of macromolecules and electron density maps. Protein Sci. 2018;27(1):86-94.

96. Hult K, Berglund P. Enzyme promiscuity: mechanism and applications. Trends Biotechnol. 2007;25:231-8.

97. Placzek S, Schomburg I, Chang A, Jeske L, Ulbrich M, Tillack J. Schomburg D. BRENDA in 2017: new perspectives and new tools in BRENDA. Nucleic Acids Res. 2017;45:D380-d388.
98. Caspi R, Altman T, Dreher K, Fulcher CA, Subhraveti P, Keseler IM, Kothari A, Krummenacker M, Latendresse M, Mueller LA, et al. The MetaCyc database of metabolic pathways and enzymes and the BioCyc collection of pathway/ genome databases. Nucleic Acids Res. 2012;40:D742-53.

99. Consortium UP. UniProt: a hub for protein information. Nucleic Acids Res. 2015:43:D204-12.

100. Katoh K, Kuma K, Toh H. Miyata T. MAFFT version 5: improvement in accuracy of multiple sequence alignment. Nucleic Acids Res. 2005;33(2):511-8.

101. Pei J, Grishin NV. PROMALS3D: multiple protein sequence alignment enhanced with evolutionary and three-dimensional structural information. Methods Mol Biol. 2014;1079:263-71.

102. Camacho C, Coulouris G, Avagyan V, Ma N, Papadopoulos J, Bealer K, Madden TL. BLAST+: architecture and applications. BMC Bioinformatics. 2009;10:421

103. Gouy M, Guindon S, Gascuel O. SeaView version 4: a multiplatform graphical user interface for sequence alignment and phylogenetic tree building. Mol Biol Evol. 2010;27(2):221-4

104. Capella-Gutierrez S, Silla-Martinez JM, Gabaldon T. trimAl: a tool for automated alignment trimming in large-scale phylogenetic analyses. Bioinformatics. 2009:25(15):1972-3.

105. Gascuel O. BIONJ: an improved version of the NJ algorithm based on a simple model of sequence data. Mol Biol Evol. 1997:14(7):685-95.

106. Price MN, Dehal PS, Arkin AP. FastTree 2--approximately maximumlikelihood trees for large alignments. PLoS One. 2010:5(3):e9490.

107. Stamatakis A. RAxML version 8: a tool for phylogenetic analysis and postanalysis of large phylogenies. Bioinformatics. 2014;30(9):1312-3.

108. Han MV, Zmasek CM. phyloXML: XML for evolutionary biology and comparative genomics. BMC Bioinformatics. 2009;10:356.

109. Rose PW, Prlic A, Altunkaya A, Bi C, Bradley AR, Christie CH, Costanzo LD, Duarte JM, Dutta S, Feng Z, et al. The RCSB protein data bank: integrative view of protein. gene and 3D structural information Nucleic Acids Res. 2017 45:D271-81

110. Cock PJ, Antao T, Chang JT, Chapman BA, Cox CJ, Dalke A, Friedberg I, Hamelryck T, Kauff F, Wilczynski B, et al. Biopython: freely available Python tools for computational molecular biology and bioinformatics. Bioinformatics. 2009;25(11):1422-3.

111. Schrödinger L. The PyMOL molecular graphics system. Version. 2:0.

112. Letunic I, Bork P. Interactive tree of life (iTOL) v3: an online tool for the display and annotation of phylogenetic and other trees. Nucleic Acids Res. 2016;44(W1):W242-5.

113. Kanehisa M, Goto S. KEGG: Kyoto encyclopedia of genes and genomes. Nucleic Acids Res. 2000:28(1):27-30.

Ready to submit your research? Choose BMC and benefit from

- fast, convenient online submission

- thorough peer review by experienced researchers in your field

- rapid publication on acceptance

- support for research data, including large and complex data types

- gold Open Access which fosters wider collaboration and increased citations

- maximum visibility for your research: over $100 \mathrm{M}$ website views per year

At BMC, research is always in progress.

Learn more biomedcentral.com/submission 